\title{
Turing-Hopf bifurcation of a ratio-dependent predator-prey model with diffusion
}

\author{
Qiushuang Shi ${ }^{1}$, Ming Liu' ${ }^{1 *}$ and Xiaofeng $\mathrm{Xu}^{1}$
}

\section{"Correspondence:}

liuming_girl@163.com

'Department of Mathematics,

Northeast Forestry University,

Harbin, P.R. China

\begin{abstract}
In this paper, the Turing-Hopf bifurcation of a ratio-dependent predator-prey model with diffusion and Neumann boundary condition is considered. Firstly, we present a kind of double parameters selection method, which can be used to analyze the Turing-Hopf bifurcation of a general reaction-diffusion equation under Neumann boundary condition. By analyzing the distribution of eigenvalues, the stable region, the unstable region (including Turing unstable region), and Turing-Hopf bifurcation point are derived in a double parameters plane. Secondly, by applying this method, the Turing-Hopf bifurcation of a ratio-dependent predator-prey model with diffusion is investigated. Finally, we compute normal forms near Turing-Hopf singularity and verify the theoretical analysis by numerical simulations.
\end{abstract}

Keywords: Ratio-dependent; Reaction-diffusion; Turing-Hopf bifurcation; Predator-prey model

\section{Introduction}

Due to the existence of rich dynamics, predator-prey systems have received great attention [1-12]. The classical ratio-dependent Holling-Tanner prey-predator model is as follows [13]:

$$
\begin{aligned}
& \frac{d u}{d t}=r u\left(1-\frac{u}{k}\right)-\frac{a u v}{m v+u}, \\
& \frac{d v}{d t}=s v\left(1-h \frac{v}{u}\right) .
\end{aligned}
$$

Here, $u$ and $v$ is the prey and predator population, respectively, $r, s>0$ is the linear birth rate of prey and predator, respectively, $k>0$ is the carrying capacity of prey population, $h>0$ is the proportionality coefficient of prey density to the carrying capacity for the predator and $\frac{a u v}{m v+u}$ represents ratio-dependent functional response with $a, m>0$, which is significant for describing predator consumption of predator-prey models [14-21].

Taking into account the inhomogeneous distribution of the prey and predators in different spatial locations and other food sources of predators, model (1) can be modified as

(c) The Author(s) 2019. This article is distributed under the terms of the Creative Commons Attribution 4.0 International License (http://creativecommons.org/licenses/by/4.0/), which permits unrestricted use, distribution, and reproduction in any medium, provided you give appropriate credit to the original author(s) and the source, provide a link to the Creative Commons license, and indicate if changes were made. 
follows [22-24]:

$$
\begin{aligned}
& \frac{\partial u}{\partial t}=d_{1} \Delta u+u\left(\alpha_{1}-\beta_{1} u-\frac{\gamma_{1} v}{m_{1} v+u}\right), \\
& \frac{\partial v}{\partial t}=d_{2} \Delta v+v\left(\alpha_{2}-\frac{\gamma_{2} v}{m_{2}+u}\right),
\end{aligned}
$$

where $u=u(x, t)$ and $v=v(x, t)$ is the population density of the prey and predators at location $x$ and time $t$, respectively, $d_{1}, d_{2}>0$ is the diffusion coefficient characterizing the rate of the spatial dispersion of the prey and predator population, respectively.

In this paper, we investigate the Turing-Hopf bifurcation of model (2) with Neumann boundary condition

$$
\frac{\partial u}{\partial v}=\frac{\partial v}{\partial v}=0, \quad x \in \partial \Omega, t>0
$$

where $\Omega=(0, l \pi)$ and $v$ is the outward unit normal vector on $\partial \Omega$. Because stable spatially inhomogeneous periodic solution can be preferably used to explain the periodic fluctuation of biological populations and it is very difficult to obtain stable spatially inhomogeneous periodic solution for research on general Turing or Hopf bifurcation under Neumann boundary condition, more and more scholars start to investigate the high codimension bifurcation of reaction-diffusion equation, especially Turing-Hopf bifurcation. There exist very rich dynamics near Turing-Hopf singularity, such as stable constant steady state, nonconstant steady state, spatially homogeneous, and inhomogeneous periodic solutions.

It is well known that the normal forms theory plays a very important role in the bifurcation analysis. Faria developed a method to calculate normal forms near an equilibrium of partial functional differential equation [25]. Based on the method of Faria, Song et al. presented a method to compute normal forms near Turing-Hopf singularity of reactiondiffusion equation [26]. However, there are still very few studies on Turing-Hopf bifurcation of reaction-diffusion equation with practical significance [27-29].

We would like to mention that one of the most difficult problems for research on TuringHopf bifurcation is how to obtain the existence of Turing-Hopf bifurcation. In the previous research, scholars generally chose two appropriate bifurcation parameters such that the Hopf and Turing bifurcation line in a double parameters plane can be defined by a straight line. This method can be easily used to obtain the existence of Turing-Hopf bifurcation, but it cannot be applied to most reaction-diffusion equations. In this paper, we present a kind of parameter selection method such that the Turing bifurcation line can be defined by a curve, which can be applied to most reaction-diffusion equations.

The rest of the paper is organized as follows. In Sect. 2, the stability of positive steady state and the existence of Turing-Hopf bifurcation for general bivariate reaction-diffusion equation are carried out. In Sect. 3, we consider Turing-Hopf bifurcation of a ratiodependent predator-prey model with diffusion by applying the results in Sect. 2. In Sect. 4, we calculate the normal forms of Turing-Hopf bifurcation for a ratio-dependent predatorprey model with diffusion. In Sect. 5 , we verify the theoretical analysis by numerical simulations. 


\section{Existence of Turing-Hopf bifurcation of reaction-diffusion equation}

In this section, we consider the Turing-Hopf bifurcation of the following general bivariate reaction-diffusion equation:

$$
\begin{cases}\frac{\partial u(x, t)}{\partial t}=d_{1} \Delta u(x, t)+\alpha F(u(x, t), v(x, t)), & x \in \Omega, t>0, \\ \frac{\partial v(x, t)}{\partial t}=d_{2} \Delta v(x, t)+\beta G(u(x, t), v(x, t)), & \\ \frac{\partial u(x, t)}{\partial v}=\frac{\partial v(x, t)}{\partial v}=0, & x \in \partial \Omega, t>0,\end{cases}
$$

where $f$ and $g$ are adequately smooth. Moreover, we assume that $\alpha, \beta>0$ and there exists one positive steady state $E_{*}\left(u_{*}, v_{*}\right)$ of system (4). Taking $\alpha$ and $\beta$ as bifurcation parameters, we have the characteristic equation at the steady state $E_{*}$ as follows:

$$
\left|\begin{array}{cc}
\lambda+d_{1}\left(\frac{n}{l}\right)^{2}-F_{1}\left(u_{*}, v_{*}\right) \alpha & -F_{2}\left(u_{*}, v_{*}\right) \alpha \\
-G_{1}\left(u_{*}, v_{*}\right) \beta & \lambda+d_{2}\left(\frac{n}{l}\right)^{2}-G_{2}\left(u_{*}, v_{*}\right) \beta
\end{array}\right|=0, \quad n \in \mathbb{N}_{0},
$$

where $\mathbb{N}_{0}=\{0\} \cup \mathbb{N}$. Denote

$$
p_{11}=F_{1}\left(u_{*}, v_{*}\right), \quad p_{12}=F_{2}\left(u_{*}, v_{*}\right), \quad p_{21}=G_{1}\left(u_{*}, v_{*}\right), \quad p_{22}=G_{2}\left(u_{*}, v_{*}\right),
$$

then Eq. (5) can be written as

$$
\Delta_{n}(\lambda)=\lambda^{2}+T_{n} \lambda+h_{n}=0, \quad n \in \mathbb{N}_{0}
$$

where

$$
\begin{aligned}
& T_{n}=\left(d_{1}+d_{2}\right)\left(\frac{n}{l}\right)^{2}+T_{0}, \\
& h_{n}=d_{1} d_{2}\left(\frac{n}{l}\right)^{4}+\left(-d_{2} p_{11} \alpha-d_{1} p_{22} \beta\right)\left(\frac{n}{l}\right)^{2}+h_{0},
\end{aligned}
$$

with

$$
\begin{aligned}
& T_{0}=-p_{11} \alpha-p_{22} \beta, \\
& h_{0}=\left(p_{11} p_{22}-p_{12} p_{21}\right) \alpha \beta .
\end{aligned}
$$

For convenience, we denote

$$
\mathcal{D}_{0}(\alpha)=-\frac{p_{11}}{p_{22}} \alpha, \quad \alpha>0
$$

and make some hypotheses as follows:

$$
\begin{aligned}
& \left(\mathrm{H}_{1}\right) \quad p_{11} p_{22}<p_{12} p_{21}, \\
& \left(\mathrm{H}_{21}\right) \quad p_{11} p_{22}>p_{12} p_{21}, \quad p_{11} \geq 0, \quad p_{22} \geq 0, \quad p_{11}^{2}+p_{22}^{2} \neq 0, \\
& \left(\mathrm{H}_{22}\right) \quad p_{11} p_{22}>p_{12} p_{21}, \quad p_{11} \leq 0, \quad p_{22} \leq 0, \quad p_{11}^{2}+p_{22}^{2} \neq 0, \\
& \left(\mathrm{H}_{23}\right) \quad p_{11} p_{22}>p_{12} p_{21}, \quad p_{11}>0, \quad p_{22}<0, \\
& \left(\mathrm{H}_{24}\right) \quad p_{11} p_{22}>p_{12} p_{21}, \quad p_{11}<0, \quad p_{22}>0 \text {. }
\end{aligned}
$$


Lemma 2.1 For system (4) without diffusion $\left(d_{1}=d_{2}=0\right)$, we have the following results.

(i) If $\left(\mathrm{H}_{1}\right)$ or $\left(\mathrm{H}_{21}\right)$ holds, then $E_{*}$ is unstable.

(ii) If $\left(\mathrm{H}_{22}\right)$ holds, then $E_{*}$ is asymptotically stable.

(iii) If $\left(\mathrm{H}_{23}\right)$ holds, then $E_{*}$ is asymptotically stable for $\beta>\mathcal{D}_{0}(\alpha)$ and unstable for $\beta<\mathcal{D}_{0}(\alpha)$; and the Hopf bifurcation line is $\beta=\mathcal{D}_{0}(\alpha)$.

(iv) If $\left(\mathrm{H}_{24}\right)$ holds, then $E_{*}$ is asymptotically stable for $\beta<\mathcal{D}_{0}(\alpha)$ and unstable for $\beta>\mathcal{D}_{0}(\alpha)$; and the Hopf bifurcation line is $\beta=\mathcal{D}_{0}(\alpha)$.

Proof Clearly, the characteristic equation for $E_{*}$ of system (4) without diffusion is

$$
\Delta_{0}(\lambda)=\lambda^{2}+T_{0} \lambda+h_{0}=0
$$

If $\left(\mathrm{H}_{1}\right)$ holds, then we have $h_{0}<0$. It follows that Eq. (11) has one positive real root and the proof of (i) is completed.

If $p_{11} p_{22}>p_{12} p_{21}$, then we have $h_{0}>0$. Furthermore, we can obtain that $T_{0}<0$ when $\left(\mathrm{H}_{21}\right)$ holds and $T_{0}>0$ when $\left(\mathrm{H}_{22}\right)$ holds. Thus, the two roots of Eq. (11) have positive real parts when $\left(\mathrm{H}_{21}\right)$ holds and negative real parts when $\left(\mathrm{H}_{22}\right)$ holds. The proof of (ii) is completed.

If $\left(\mathrm{H}_{23}\right)$ holds, we can obtain that $h_{0}>0$ and $T_{0}<0$ when $\beta<\mathcal{D}_{0}(\alpha)$ and $T_{0}>0$ when $\beta>$ $\mathcal{D}_{0}(\alpha)$. Moreover, $\pm i \sqrt{h_{0}}$ are a pair of purely imaginary roots of Eq. (11) when $\beta=\mathcal{D}_{0}(\alpha)$ and the transversality condition is as follows:

$$
\left.\frac{d \operatorname{Re}\{\lambda(\alpha)\}}{d \alpha}\right|_{\beta=\mathcal{D}_{0}(\alpha)}=\frac{p_{11}}{2}>0 .
$$

Thus, $\beta=\mathcal{D}_{0}(\alpha)$ is the Hopf bifurcation line and the proof of (iii) is completed. We omit the proof of (iv), because it is similar to the proof of (iii).

Assumptions in (10) do not include the cases $p_{11} p_{22}=p_{12} p_{21}$ and $p_{11}=p_{22}=0$, because if $p_{11} p_{22}=p_{12} p_{21}$ holds, one immediately has $h_{0}=0$ and 0 is a root of Eq. (11) for any $\alpha, \beta>0$ and if $p_{11}=p_{22}=0$ holds, we immediately have $T_{0}=0$ and $\pm i \sqrt{h_{0}}$ are a pair of purely imaginary roots of Eq. (11) for any $\alpha, \beta>0$. Obviously, the double parameters selection method is not effective for these two cases. So we do not discuss them any more in this paper.

Next, we investigate the stability and Turing-Hopf bifurcation of system (4). From the proof of Lemma 2.1, we know that Eq. (6) has at least one root with a positive real part for any $\alpha, \beta>0$ if $\left(\mathrm{H}_{1}\right)$ or $\left(\mathrm{H}_{21}\right)$ holds. Moreover, if $\left(\mathrm{H}_{22}\right)$ holds, one can easily obtain that $T_{n}>0$ and $h_{n}>0$ for $n \in \mathbb{N}_{0}$ and $\alpha, \beta>0$. Therefore, we have the following result.

\section{Theorem 2.2}

(i) If $\left(\mathrm{H}_{1}\right)$ or $\left(\mathrm{H}_{21}\right)$ holds, $E_{*}$ is unstable for any $\alpha, \beta>0$.

(ii) If $\left(\mathrm{H}_{22}\right)$ holds, $E_{*}$ is asymptotically stable for any $\alpha, \beta>0$.

Since $\left(\mathrm{H}_{24}\right)$ can be converted to $\left(\mathrm{H}_{23}\right)$ by swapping the two equations of system (4), we focus on the case of $\left(\mathrm{H}_{23}\right)$. Assume that $\left(\mathrm{H}_{23}\right)$ holds and analyze the Turing-Hopf bifurcation of system (4). First, we consider the case of no diffusion-driven Turing instability. 
If $\beta>\mathcal{D}_{0}(\alpha)$ holds, we have that $T_{n}>0$ for $n \in \mathbb{N}_{0}$. And if $h_{n}=0$ holds, we have that

$$
\beta=\mathcal{R}_{n}(\alpha)=\frac{d_{2}\left(\frac{n}{l}\right)^{2} p_{11} \alpha-d_{1} d_{2}\left(\frac{n}{l}\right)^{4}}{-d_{1}\left(\frac{n}{l}\right)^{2} p_{22}+\left(p_{11} p_{22}-p_{12} p_{21}\right) \alpha}, \quad n \in \mathbb{N}_{0}
$$

Obviously, if all lines defined by $\beta=\mathcal{R}_{n}(\alpha)$ lie below the Hopf bifurcation line $\beta=\mathcal{D}_{0}(\alpha)$, there is no Turing instability. Note that

$$
\mathcal{D}_{0}(\alpha)-\mathcal{R}_{n}(\alpha)=\frac{1}{-d_{1}\left(\frac{n}{l}\right)^{2} p_{22}+\left(p_{11} p_{22}-p_{12} p_{21}\right) \alpha} P_{n}(\alpha),
$$

where

$$
P_{n}(\alpha)=-\frac{p_{11}}{p_{22}}\left(p_{11} p_{22}-p_{12} p_{21}\right) \alpha^{2}+\left(d_{1}-d_{2}\right) p_{11}\left(\frac{n}{l}\right)^{2} \alpha+d_{1} d_{2}\left(\frac{n}{l}\right)^{4}
$$

For convenience, we define

$$
\Lambda=\left(d_{2}-d_{1}\right)^{2} p_{11}^{2}+4 d_{1} d_{2} \frac{p_{11}}{p_{22}}\left(p_{11} p_{22}-p_{12} p_{21}\right)
$$

and make some hypotheses:

$$
\begin{aligned}
& \left(\mathrm{C}_{1}\right) \quad d_{2} \leq d_{1} . \\
& \left(\mathrm{C}_{2}\right) \quad d_{2}>d_{1} \text { and } \Lambda<0 . \\
& \left(\mathrm{C}_{3}\right) \quad d_{2}>d_{1} \text { and } \Lambda>0 .
\end{aligned}
$$

Clearly, $\mathcal{D}_{0}(\alpha)>\mathcal{R}_{n}(\alpha)$ is equivalent to $P_{n}(\alpha)>0$. Moreover, we can obtain that $P_{n}(\alpha)>0$ for $n \in \mathbb{N}_{0}$ if $\left(C_{1}\right)$ or $\left(C_{2}\right)$ holds. Hence, we have the following result.

Theorem 2.3 Assume that $\left(\mathrm{H}_{23}\right)$ holds. If $\left(\mathrm{C}_{1}\right)$ or $\left(\mathrm{C}_{2}\right)$ is satisfied, then system (4) has no diffusion-driven Turing instability. In this case, diffusion does not change the stability of $E^{*}$, i.e., the stable and unstable regions are the same as those in Lemma 2.1(iii).

Then we focus on the case of the existence of Turing instability. It is easy to see that if $\left(C_{3}\right)$ holds, $\mathcal{D}_{0}(\alpha)-\mathcal{R}_{n}(\alpha)=0, n \in \mathbb{N}$ has two positive roots:

$$
\mathcal{A}_{n}^{ \pm}=\frac{-\left(d_{1}-d_{2}\right) p_{11} \pm \sqrt{\Lambda}}{-2 \frac{p_{11}}{p_{22}}\left(p_{11} p_{22}-p_{12} p_{21}\right)}\left(\frac{n}{l}\right)^{2}
$$

such that

$$
\begin{cases}\mathcal{D}_{0}(\alpha)<\mathcal{R}_{n}(\alpha) & \text { if } \mathcal{A}_{n}^{-}<\alpha<\mathcal{A}_{n}^{+} \\ \mathcal{D}_{0}(\alpha)>\mathcal{R}_{n}(\alpha) & \text { if } 0<\alpha<\mathcal{A}_{n}^{-} \text {or } \alpha>\mathcal{A}_{n}^{+}\end{cases}
$$

For any $m, n \in \mathbb{N}, m>n$, we can obtain that

$$
\mathcal{R}_{m}(\alpha)-\mathcal{R}_{n}(\alpha)
$$




$$
\begin{aligned}
& =\frac{d_{2}\left(\frac{m}{l}\right)^{2} p_{11} \alpha-d_{1} d_{2}\left(\frac{m}{l}\right)^{4}}{-d_{1}\left(\frac{m}{l}\right)^{2} p_{22}+\left(p_{11} p_{22}-p_{12} p_{21}\right) \alpha}-\frac{d_{2}\left(\frac{n}{l}\right)^{2} p_{11} \alpha-d_{1} d_{2}\left(\frac{n}{l}\right)^{4}}{-d_{1}\left(\frac{n}{l}\right)^{2} p_{22}+\left(p_{11} p_{22}-p_{12} p_{21}\right) \alpha} \\
& =\frac{d_{2}\left(m^{2}-n^{2}\right) Q_{n m}(\alpha)}{\left(-d_{1}\left(\frac{m}{l}\right)^{2} p_{22}+\left(p_{11} p_{22}-p_{12} p_{21}\right) \alpha\right)\left(-d_{1}\left(\frac{n}{l}\right)^{2} p_{22}+\left(p_{11} p_{22}-p_{12} p_{21}\right) \alpha\right) l^{2}},
\end{aligned}
$$

where

$$
Q_{n m}(\alpha)=p_{11}\left(p_{11} p_{22}-p_{12} p_{21}\right) \alpha^{2}-d_{1} \frac{m^{2}+n^{2}}{l^{2}}\left(p_{11} p_{22}-p_{12} p_{21}\right) \alpha+d_{1}^{2} \frac{m^{2} n^{2}}{l^{4}} p_{22} .
$$

Note that, for any $m, n \in \mathbb{N}, Q_{n m}(\alpha)$ has and only has one positive root

$$
\mathcal{B}^{+}(n, m)=\frac{d_{1}\left(m^{2}+n^{2}\right)}{2 p_{11} l^{2}}+\sqrt{\frac{d_{1}^{2}\left(m^{2}+n^{2}\right)^{2}}{4 p_{11}^{2} l^{4}}-\frac{d_{1}^{2} p_{22} m^{2} n^{2}}{p_{11}\left(p_{11} p_{22}-p_{12} p_{21}\right) l^{4}}}
$$

such that

$$
\begin{cases}\mathcal{R}_{m}(\alpha)<\mathcal{R}_{n}(\alpha) & \text { if } 0<\alpha<\mathcal{B}^{+}(n, m) \\ \mathcal{R}_{m}(\alpha)>\mathcal{R}_{n}(\alpha) & \text { if } \alpha>\mathcal{B}^{+}(n, m)\end{cases}
$$

Therefore, $\beta=\mathcal{R}_{m}(\alpha)$ and $\beta=\mathcal{R}_{n}(\alpha)$ have and only have one intersecting point for any $m, n \in \mathbb{N}, m>n$. By (14) and (15), we can obtain that $\mathcal{A}_{n}^{ \pm}$is monotonously increasing with respect to $n$ and $\mathcal{B}^{+}(n, m)$ is monotonously increasing with respect to $m$ for fixed $n \in \mathbb{N}$.

Moreover, the transversality condition is as follows:

$$
\left.\frac{d \operatorname{Re}\{\lambda(\alpha)\}}{d \alpha}\right|_{\beta=\mathcal{R}_{n}(\alpha)}=\frac{d_{1} d_{2}\left(\frac{n}{l}\right)^{4}-d_{1} p_{22} \beta\left(\frac{n}{l}\right)^{2}}{T_{n} \alpha}>0 \quad \text { if } \beta>\mathcal{D}_{0}(\alpha) .
$$

For convenience, denote

$$
\Gamma_{0}=\mathcal{A}_{1}^{-}, \Gamma_{n}= \begin{cases}\mathcal{B}^{+}(n, n+1) & \text { if } \mathcal{A}_{n+1}^{-} \leq \mathcal{A}_{n}^{+}, \quad n \in \mathbb{N}, \\ \mathcal{A}_{n+1}^{-} & \text {if } \mathcal{A}_{n+1}^{-}>\mathcal{A}_{n}^{+},\end{cases}
$$

and

$$
\begin{aligned}
& \mathcal{T}_{0}(\alpha)=\mathcal{D}_{0}(\alpha), \quad \alpha \in\left(0, \Gamma_{0}\right], \\
& \mathcal{T}_{n}(\alpha)=\left\{\begin{array}{ll}
\mathcal{R}_{n}(\alpha) & \text { if } \mathcal{A}_{n+1}^{-} \leq \mathcal{A}_{n}^{+}, \\
\tilde{\mathcal{T}}_{n}(\alpha) & \text { if } \mathcal{A}_{n+1}^{-}>\mathcal{A}_{n}^{+},
\end{array} \quad n \in \mathbb{N}, \alpha \in\left(\Gamma_{n-1}, \Gamma_{n}\right],\right.
\end{aligned}
$$

with

$$
\tilde{\mathcal{T}}_{n}(\alpha)=\left\{\begin{array}{ll}
\mathcal{R}_{n}(\alpha), & \alpha \in\left(\Gamma_{n-1}, \mathcal{A}_{n}^{+}\right], \\
\mathcal{D}_{0}(\alpha), & \alpha \in\left(\mathcal{A}_{n}^{+}, \Gamma_{n}\right],
\end{array} \quad n \in \mathbb{N} .\right.
$$

It is clear that for $0<\alpha \leq \Gamma_{0}=\mathcal{A}_{1}^{-}$, the boundary of an unstable region of $E_{*}$ is $\mathcal{D}_{0}(\alpha)$. If $\mathcal{A}_{2}^{-} \leq \mathcal{A}_{1}^{+}$, then for $\Gamma_{0}<\alpha \leq \Gamma_{1}=\mathcal{B}^{+}(1,2)$, the boundary of a stable region is $\mathcal{R}_{1}(\alpha)$. 
Otherwise, for $\Gamma_{0}<\alpha \leq \Gamma_{1}=\mathcal{A}_{2}^{-}$, the boundaries consist of $\mathcal{R}_{1}(\alpha)$ with $\Gamma_{0}<\alpha \leq \mathcal{A}_{1}^{+}$and $\mathcal{D}_{0}(\alpha)$ with $\mathcal{A}_{1}^{+}<\alpha \leq \Gamma_{1}$. By using the mathematical induction, we can obtain that all the boundaries of a stable region consist of $\mathcal{T}_{n}(\alpha), n \in \mathbb{N}_{0}$.

Obviously, when $(\alpha, \beta)=\left(\mathcal{A}_{1}^{-},-\frac{p_{11}}{p_{22}} \mathcal{A}_{1}^{-}\right), \Delta_{0}(\lambda)=0$ has a pair of purely imaginary roots $\pm i \sqrt{h_{0}}$ and $\Delta_{1}(\lambda)=0$ has a root $\lambda=0$ with a negative real root $\lambda=-T_{1}$ and all other roots have negative real parts. Together with the transversality conditions (12) and (18), we have that system (4) undergoes Turing-Hopf bifurcation at $\left(\mathcal{A}_{1}^{-},-\frac{p_{11}}{p_{22}} \mathcal{A}_{1}^{-}\right)$. If $\mathcal{A}_{n+1}^{-}>\mathcal{A}_{n}^{+}$holds, $\Delta_{n}(\lambda)=0$ and $\Delta_{n+1}(\lambda)=0$ have zero roots under the conditions $(\alpha, \beta)=\left(\mathcal{A}_{n}^{+},-\frac{p_{11}}{p_{22}} \mathcal{A}_{n}^{+}\right)$and $(\alpha, \beta)=\left(\mathcal{A}_{n+1}^{-},-\frac{p_{11}}{p_{22}} \mathcal{A}_{n+1}^{-}\right)$, respectively. Meanwhile all other roots, except a pair of purely imaginary roots $\pm i \sqrt{h_{0}}$ of $\Delta_{0}(\lambda)=0$, have negative real parts. Thus, $\left(\mathcal{A}_{n}^{+},-\frac{p_{11}}{p_{22}} \mathcal{A}_{n}^{+}\right)$and $\left(\mathcal{A}_{n+1}^{-},-\frac{p_{11}}{p_{22}} \mathcal{A}_{n+1}^{-}\right)$are in the same situation.

From the above analysis, we can detailedly describe the boundary of the unstable region (including Turing unstable region) of the positive steady state $E_{*}$.

Theorem 2.4 Assume that $\left(\mathrm{H}_{23}\right)$ holds and $\left(\mathrm{C}_{3}\right)$ is satisfied, there exists diffusion-driven Turing instability. More precisely, we have the following results.

(i) The boundary of a stable region is $\beta=\mathcal{T}_{n}(\alpha), n \in \mathbb{N}_{0}$, and for any $\alpha>0, \beta>\mathcal{T}_{n}(\alpha)$, $n \in \mathbb{N}_{0}$, the positive steady state $E_{*}$ is asymptotically stable.

(ii) For any $\alpha>0, \mathcal{D}_{0}(\alpha)<\beta<\mathcal{T}_{n}(\alpha), n \in \mathbb{N}$, there exists Turing instability.

(iii) System (4) undergoes Turing-Hopf bifurcation at the point $\left(\mathcal{A}_{1}^{-},-\frac{p_{11}}{p_{22}} \mathcal{A}_{1}^{-}\right)$; if $\mathcal{A}_{n+1}^{-}>\mathcal{A}_{n}^{+}, n \in \mathbb{N}$, holds, system (4) undergoes Turing-Hopf bifurcation at the points $\left(\mathcal{A}_{n}^{+},-\frac{p_{11}}{p_{22}} \mathcal{A}_{n}^{+}\right)$and $\left(\mathcal{A}_{n+1}^{-},-\frac{p_{11}}{p_{22}} \mathcal{A}_{n+1}^{-}\right)$.

\section{Turing-Hopf bifurcation of a diffusive ratio-dependent predator-prey model}

Let

$$
h=\frac{\beta}{\alpha_{1}}, \quad \delta=\frac{\gamma_{1}}{\alpha_{1}}, \quad b=\frac{\gamma_{2}}{\alpha_{2}},
$$

then system (2) becomes:

$$
\left\{\begin{array}{lll}
\frac{\partial u(x, t)}{\partial t}=d_{1} \Delta u(x, t)+\alpha_{1} u(x, t)\left(1-h u(x, t)-\frac{\delta v(x, t)}{m_{1} v(x, t)+u(x, t)}\right), & & x \in \Omega, t>0, \\
\frac{\partial v(x, t)}{\partial t}=d_{2} \Delta v(x, t)+\alpha_{2} v(x, t)\left(1-\frac{b v(x, t)}{m_{2}+u(x, t)}\right), & & x \in \partial \Omega, t>0 . \\
\frac{\partial u(x, t)}{\partial v}=\frac{\partial v(x, t)}{\partial v}=0, &
\end{array}\right.
$$

Next, we use the conclusions of the above section to investigate the stability of positive steady state and Turing-Hopf bifurcation of system (23). With a simple calculation, we have the following result.

Theorem 3.1 Assume that $\alpha_{1}, \alpha_{2}, d_{1}, d_{2}, l>0$. Then the existence conditions of positive steady state of model (23) can be divided into the following three situations:

Case $A: m_{1}=\delta$ and $b>h m_{1} m_{2}+1$.

There exists only one positive steady state $E_{1}=\left(u_{1}, v_{1}\right)$ of model (23) in this case, where

$$
u_{1}=\frac{b-h m_{1} m_{2}}{h\left(m_{1}+b\right)}, \quad v_{1}=\frac{m_{2}+u_{1}}{b}
$$


Case $B: m_{1}>\delta$.

There exists only one positive steady state $E_{2}=\left(u_{2}, v_{2}\right)$ of model (23) in this case, where

$$
u_{2}=\frac{m_{1}+b-h m_{1} m_{2}-\delta+c}{2 h\left(m_{1}+b\right)}, \quad v_{2}=\frac{m_{2}+u_{2}}{b},
$$

with

$$
c=\sqrt{\left(m_{1}+b-h m_{1} m_{2}-\delta\right)^{2}+4 h m_{2}\left(m_{1}+b\right)\left(m_{1}-\delta\right)} .
$$

Case $C: m_{1}<\delta$ and $m_{1}+b-h m_{1} m_{2}-\delta>0$.

There exist two positive steady states $E_{3}=\left(u_{3}, v_{3}\right)$ and $E_{4}=\left(u_{4}, v_{4}\right)$ of model (23) in this case, where

$$
\begin{array}{ll}
u_{3}=\frac{m_{1}+b-h m_{1} m_{2}-\delta-c}{2 h\left(m_{1}+b\right)}, & v_{3}=\frac{m_{2}+u_{3}}{b}, \\
u_{4}=\frac{m_{1}+b-h m_{1} m_{2}-\delta+c}{2 h\left(m_{1}+b\right)}, & v_{4}=\frac{m_{2}+u_{4}}{b} .
\end{array}
$$

Next, we take the steady state $E_{4}$ as an example to investigate the Turing-Hopf bifurcation. Denote

$$
\begin{aligned}
& f\left(u_{4}\right)=\frac{\delta m_{1}\left(m_{2}+u_{4}\right)^{2}}{\left(m_{1}\left(m_{2}+u_{4}\right)+b u_{4}\right)^{2}}, \\
& g\left(u_{4}\right)=\frac{b^{2} u_{4}^{2} \delta}{\left(m_{1}\left(m_{2}+u_{4}\right)+b u_{4}\right)^{2}}, \\
& r\left(u_{4}\right)=1-2 h u_{4}-f\left(u_{4}\right) .
\end{aligned}
$$

Then we have

$$
p_{11}=r\left(u_{4}\right), \quad p_{12}=-g\left(u_{4}\right), \quad p_{21}=\frac{1}{b}, \quad p_{22}=-1 .
$$

The characteristic equation at $E_{4}$ is

$$
\Delta_{n}(\lambda)=\lambda^{2}+T_{n} \lambda+h_{n}=0, \quad n \in \mathbb{N}_{0}
$$

where

$$
\begin{aligned}
& T_{n}=\left(d_{1}+d_{2}\right)\left(\frac{n}{l}\right)^{2}+T_{0}, \\
& h_{n}=d_{1} d_{2}\left(\frac{n}{l}\right)^{4}+\left(d_{1} \alpha_{2}-d_{2} \alpha_{1} r\left(u_{4}\right)\right)\left(\frac{n}{l}\right)^{2}+h_{0},
\end{aligned}
$$

with

$$
\begin{aligned}
& T_{0}=\alpha_{2}-\alpha_{1} r\left(u_{4}\right), \\
& h_{0}=\alpha_{1} \alpha_{2} \frac{g\left(u_{4}\right)}{b}-\alpha_{1} \alpha_{2} r\left(u_{4}\right) .
\end{aligned}
$$


From the results in Sect. 2, one immediately has the following conclusions.

\section{Theorem 3.2}

(i) If $r\left(u_{4}\right) \leq 0$ holds, the positive steady state $E_{4}$ of (23) is asymptotically stable for any $\alpha_{1}, \alpha_{2}>0$.

(ii) If $r\left(u_{4}\right)>0$ and $\frac{g\left(u_{4}\right)}{b}<r\left(u_{4}\right)$ hold, the positive steady state $E_{4}$ of (23) is unstable for any $\alpha_{1}, \alpha_{2}>0$.

Theorem 3.3 Suppose that $r\left(u_{4}\right)>0, \frac{g\left(u_{4}\right)}{b}>r\left(u_{4}\right)$. If $\left(\mathrm{C}_{1}\right)$ or $\left(\mathrm{C}_{2}\right)$ holds, there is no diffusion-driven Turing instability.

Theorem 3.4 Suppose that $r\left(u_{4}\right)>0, \frac{g\left(u_{4}\right)}{b}>r\left(u_{4}\right)$. If $\left(C_{3}\right)$ holds, we have the following results.

(i) If $\alpha_{1}>0, \alpha_{2}>\mathcal{T}_{n}\left(\alpha_{1}\right), n \in \mathbb{N}_{0}$, the steady state $E_{4}$ is asymptotically stable.

(ii) Turing instability occurs for $\alpha_{1}>0, \mathcal{D}_{0}\left(\alpha_{1}\right)<\alpha_{2}<\mathcal{T}_{n}\left(\alpha_{1}\right), n \in \mathbb{N}$.

(iii) System (23) undergoes Turing-Hopf bifurcation at $\left(\mathcal{A}_{1}^{-}, r\left(u_{4}\right) \mathcal{A}_{1}^{-}\right)$; if $\mathcal{A}_{n+1}^{-}>\mathcal{A}_{n}^{+}, n \in \mathbb{N}$ holds, system (23) undergoes Turing-Hopf bifurcation at the point $\left(\mathcal{A}_{n}^{+}, r\left(u_{4}\right) \mathcal{A}_{n}^{+}\right)$and $\left(\mathcal{A}_{n+1}^{-}, r\left(u_{4}\right) \mathcal{A}_{n+1}^{-}\right)$, where

$$
\begin{aligned}
& \mathcal{D}_{0}\left(\alpha_{1}\right)=\alpha_{1} r\left(u_{4}\right), \quad \alpha_{1}>0, \\
& \mathcal{R}_{n}\left(\alpha_{1}\right)=\frac{r\left(u_{4}\right) \alpha_{1} d_{2}\left(\frac{n}{l}\right)^{2}-d_{1} d_{2}\left(\frac{n}{l}\right)^{4}}{d_{1}\left(\frac{n}{l}\right)^{2}+\alpha_{1}\left(\frac{g\left(u_{4}\right)}{b}-r\left(u_{4}\right)\right)}, \quad r>0, n \in \mathbb{N}_{0} . \\
& \Lambda=r^{2}\left(u_{4}\right)\left(d_{2}-d_{1}\right)^{2}-4 r\left(u_{4}\right)\left(\frac{g\left(u_{4}\right)}{b}-r\left(u_{4}\right)\right) d_{1} d_{2}, \\
& \mathcal{A}_{n}^{ \pm}=\frac{r\left(u_{4}\right)\left(d_{2}-d_{1}\right) \pm \sqrt{\Lambda}}{2 r\left(u_{4}\right)\left(\frac{g\left(u_{4}\right)}{b}-r\left(u_{4}\right)\right)}\left(\frac{n}{l}\right)^{2}, \\
& \mathcal{B}^{+}(n, m)=\frac{d_{1}\left(\frac{g\left(u_{4}\right)}{b}-r\left(u_{4}\right)\right) \frac{m^{2}+n^{2}}{l^{2}}+c_{1}}{2 r\left(u_{4}\right)\left(\frac{g\left(u_{4}\right)}{b}-r\left(u_{4}\right)\right)}, \\
& c_{1}=\sqrt{d_{1}^{2}\left(\frac{g\left(u_{4}\right)}{b}-r\left(u_{4}\right)\right)^{2} \frac{\left(m^{2}+n^{2}\right)^{2}}{l^{2}}+4 d_{1}^{2} r\left(u_{4}\right)\left(\frac{g\left(u_{4}\right)}{b}-r\left(u_{4}\right)\right) \frac{m^{2} n^{2}}{l^{4}} .}
\end{aligned}
$$

$\Gamma_{n}$ and $\mathcal{T}_{n}$ are defined by (21) and (22), respectively, and hypotheses $\left(C_{1}\right),\left(C_{2}\right)$, and $\left(C_{3}\right)$ are defined by (17).

We would like to mention that $\mathcal{A}_{n+1}^{-}<\mathcal{A}_{n}^{+}$and $\mathcal{A}_{n+1}^{-}>\mathcal{A}_{n}^{+}$are all possible to happen (see Fig. 1). Obviously, we have $\mathcal{A}_{2}^{-}<\mathcal{A}_{1}^{+}$in Fig. 1(a) and $\mathcal{A}_{2}^{-}>\mathcal{A}_{1}^{+}$in Fig. 1(b).

\section{Normal forms for Turing-Hopf bifurcation}

From Theorem 3.4, we know that for given appropriate parameters, there is at least one Turing-Hopf bifurcation point in the $\left(\alpha_{1}, \alpha_{2}\right)$ plane. Denote the Turing-Hopf bifurcation point (an intersecting point of the lines $\alpha_{2}=\mathcal{D}_{0}\left(\alpha_{1}\right)$ and $\left.\alpha_{2}=\mathcal{R}_{n_{*}}\left(\alpha_{1}\right)\right)$ as $\left(\alpha_{1 *}, \alpha_{2 *}\right)$, where $\alpha_{2 *}=r\left(u_{4}\right) \alpha_{1 *}$. Next we compute the normal forms for Turing-Hopf bifurcation by using the method in [26]. 


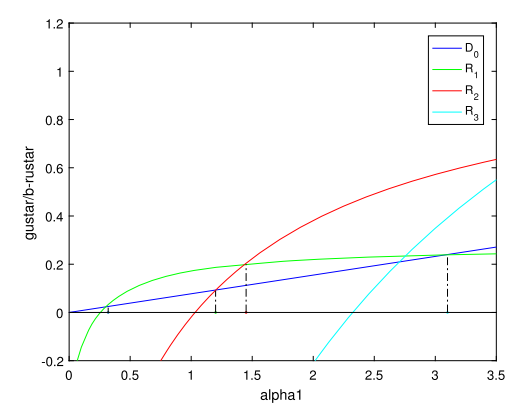

(a)

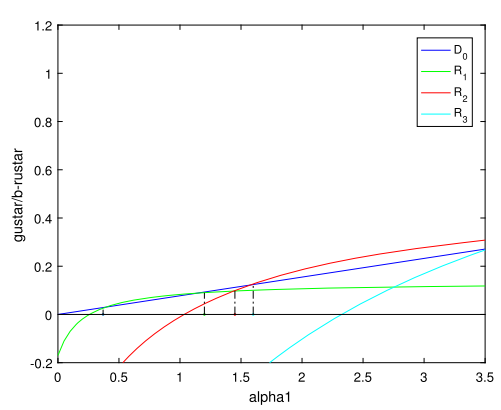

(b)

Figure 1 Bifurcation diagram in the double parameters plane at the positive steady state $E_{4}$, where $b=0.35, \delta=0.6, m_{1}=0.5, m_{2}=0.3, h=0.3, I=1, d_{1}=0.02 .(\mathbf{a}): d_{2}=0.35 .(\mathbf{b}): d_{2}=0.17$

Let $\alpha_{1}=\alpha_{1 *}+\mu_{1}$ and $\alpha_{2}=\alpha_{2 *}+\mu_{2}$, then system (23) becomes

$$
\left\{\begin{array}{l}
\frac{\partial u}{\partial t}=d_{1} \Delta u+\left(\alpha_{1 *}+\mu_{1}\right) u\left(1-h u-\frac{\delta v}{m_{1} v+u}\right) \\
\frac{\partial v}{\partial t}=d_{2} \Delta v+\left(\alpha_{2 *}+\mu_{2}\right) v\left(1-\frac{b v}{m_{2}+u}\right)
\end{array}\right.
$$

Clearly, $\left(\mu_{1}, \mu_{2}\right)=(0,0)$ is the Turing-Hopf bifurcation point of system (25). Set $\bar{u}=u-u_{4}$ and $\bar{v}=v-\frac{m_{2}+u_{4}}{b}$. Then system (25) becomes

$$
\left\{\begin{array}{l}
\frac{\partial u}{\partial t}=d_{1} \Delta u+\left(\alpha_{1 *}+\mu_{1}\right)\left(u+u_{4}\right)\left(1-h\left(u+u_{4}\right)-\frac{\delta\left(v+\frac{m_{2}+u_{4}}{b}\right)}{m_{1}\left(v+\frac{m_{2}+u_{4}}{b}\right)+\left(u+u_{4}\right)},\right. \\
\frac{\partial v}{\partial t}=d_{2} \Delta v+\left(\alpha_{2 *}+\mu_{2}\right)\left(v+\frac{m_{2}+u_{4}}{b}\right)\left(1-\frac{b\left(v+\frac{m_{2}+u_{4}}{b}\right)}{m_{2}+\left(u+u_{4}\right)}\right)
\end{array}\right.
$$

Define the real-valued Sobolev space

$$
\mathscr{X}=\left\{(u, v) \in H^{2}(0, l \pi), \frac{\partial u}{\partial t}=\frac{\partial v}{\partial t}=0 \text { at } x=0, l \pi\right\}
$$

with the inner product

$$
[U, V]=\int_{0}^{l \pi}\left(u_{1} v_{1}+u_{2} v_{2}\right) d x, \quad \text { for } U=\left(u_{1}, u_{2}\right)^{T}, V=\left(v_{1}, v_{2}\right)^{T} \in \mathscr{X}_{1} .
$$

In the abstract space $\mathcal{C}=C(\mathbb{R}, \mathscr{X})$, system (26) can be written as

$$
\dot{U}=\mathscr{L} U+\tilde{F}(U, \mu)
$$

where $U=(u, v)^{T}, \mu=\left(\mu_{1}, \mu_{2}\right), \mathscr{L} U=D \Delta U+L_{0}(U), D=\operatorname{diag}\left(d_{1}, d_{2}\right), \tilde{F}(U, \mu)=L(\mu)(U)-$ $L_{0}(U)+F(U, \mu)$ with $L_{0}=L(0)$ and

$$
\begin{aligned}
& L(\mu)=\left(\begin{array}{cc}
r\left(u_{4}\right)\left(\alpha_{1 *}+\mu_{1}\right) & -\left(\alpha_{1 *}+\mu_{1}\right) g\left(u_{4}\right) \\
\frac{\alpha_{2 *}+\mu_{2}}{b} & -\left(\alpha_{2 *}+\mu_{2}\right)
\end{array}\right), \\
& F(U, \mu)=\left(\begin{array}{l}
F^{(1)}\left(u, v, \mu_{1}, \mu_{2}\right) \\
F^{(2)}\left(u, v, \mu_{1}, \mu_{2}\right)
\end{array}\right)
\end{aligned}
$$




$$
=\left(\begin{array}{c}
\left(\alpha_{1 *}+\mu_{1}\right)\left(-h u^{2}-\frac{\delta\left(v+\frac{m_{2}+u_{4}}{b}\right)\left(u+u_{4}\right)}{m_{1}\left(v+\frac{m_{2}+u_{4}}{b}\right)+\left(u+u_{4}\right)}+u_{4}-h u_{4}^{2}\right) \\
-\left(\alpha_{1 *}+\mu_{1}\right) f\left(u_{4}\right) u+\left(\alpha_{1 *}+\mu_{1}\right) g\left(u_{4}\right) v \\
\left(\alpha_{2 *}+\mu_{2}\right)\left(v+\frac{m_{2}+u_{4}}{b}\right)\left(1-\frac{b\left(v+\frac{m_{2}+u_{4}}{b}\right)}{m_{2}+\left(u+u_{4}\right)}\right)+\left(\alpha_{2 *}+\mu_{2}\right) v \\
-\frac{\left(\alpha_{2 *}+\mu_{2}\right) u}{b}
\end{array}\right) .
$$

Denote the eigenvalues of $D \Delta$ on $\mathscr{X}$ by

$$
\delta_{n}^{(j)}=-d_{j}\left(\frac{n}{l}\right)^{2}, \quad j=1,2, n \in \mathbb{N}_{0}
$$

and the corresponding normalized eigenfunctions by

$$
\beta_{n}^{(j)}=\gamma_{n}(x) e_{j}, \quad \gamma_{n}(x)=\frac{\cos \left(\frac{n x}{l}\right)}{\left\|\cos \left(\frac{n x}{l}\right)\right\|_{2,2}}= \begin{cases}\frac{1}{\sqrt{l \pi}} & \text { for } n=0, \\ \frac{\sqrt{2}}{\sqrt{l \pi}} \cos \left(\frac{n x}{l}\right) & \text { for } n \neq 0,\end{cases}
$$

where $e_{j}$ is the unit coordinate vector of $\mathbb{R}^{2}$ ( $n$ is wave number).

By the Taylor expansions of $L(\mu)$, we have that

$$
L(\mu)=L_{0}+\mu_{1} L_{1}^{(1,0)}+\mu_{2} L_{1}^{(0,1)}+\frac{1}{2}\left(\mu_{1}^{2} L_{2}^{(2,0)}+2 \mu_{1} \mu_{2} L_{2}^{(1,1)}+\mu_{2}^{2} L_{2}^{(0,2)}\right)+\cdots
$$

where

$$
\begin{aligned}
& L_{0}=\left(\begin{array}{cc}
\alpha_{1 *} r\left(u_{4}\right) & -\alpha_{1 *} g\left(u_{4}\right) \\
\frac{\alpha_{2 *}}{b} & -\alpha_{2 *}
\end{array}\right), \quad L_{1}^{(1,0)}=\left(\begin{array}{cc}
r\left(u_{4}\right) & -g\left(u_{4}\right) \\
0 & 0
\end{array}\right), \\
& L_{1}^{(0,1)}=\left(\begin{array}{cc}
0 & 0 \\
\frac{1}{b} & -1
\end{array}\right) .
\end{aligned}
$$

Let

$$
F_{j_{1} j_{2}}=\left(F_{j_{1} j_{2}}^{(1)}, F_{j_{1} j_{2}}^{(2)}\right)^{T}
$$

with

$$
F_{j_{1} j_{2}}^{(k)}=\frac{\partial^{j_{1}+j_{2}} F^{(k)}(0,0,0,0)}{\partial w^{j_{1}} \partial v^{j_{2}}}, \quad k=1,2, j_{1}+j_{2}=2,3 .
$$

Then from (28) we have

$$
\begin{aligned}
& F_{20}=\left(\begin{array}{c}
\left.-2 h-\frac{2 \delta u_{*} \frac{m_{2}+u_{*}}{b}}{\left(u_{*}+m 1 \frac{m_{2}+u_{*}}{b}\right)^{3}}+\frac{2 \delta \frac{m_{2}+u_{*}}{b}}{\left(u_{*}+m 1 \frac{m_{2}+u_{*}}{b}\right)^{2}}\right), \\
\frac{-2 \alpha_{2 *}}{b\left(m_{2}+u_{*}\right)}
\end{array}\right), \\
& F_{02}=\left(\begin{array}{c}
-\frac{2 \delta m_{1} u_{*}}{\left(u_{*}+m 1 \frac{m_{2}+u_{*}}{b}\right)^{2}}-\frac{2 \delta m_{1}^{2} \frac{m_{2}+u_{*}}{b}}{\left(u_{*}+m 1 \frac{m_{2}+u_{*}}{b}\right)^{3}} \\
-\frac{-2 \alpha_{2 *} b}{m_{2}+u_{*}}
\end{array}\right), \\
& F_{30}=\left(\begin{array}{c}
\frac{6 \delta u_{*} \frac{m_{2}+u_{*}}{b}}{\left(u_{*}+m 1 \frac{m_{2}+u_{*}}{b}\right)^{4}}-\frac{6 \delta \frac{m_{2}+u_{*}}{b}}{\left(u_{*}+m 1 \frac{m_{2}+u_{*}}{b}\right)^{3}} \\
\frac{6 \alpha_{2 *}}{b\left(m_{2}+u_{*}\right)^{2}}
\end{array}\right),
\end{aligned}
$$




$$
F_{03}=\left(\begin{array}{c}
-\frac{6 \delta m_{1}^{2} u_{*}}{\left(u_{*}+m 1 \frac{m_{2}+u_{*}}{b}\right)^{2}}+\frac{6 \delta m_{1}^{3} u_{*} \frac{m_{2}+u_{*}}{b}}{\left(u_{*}+m 1 \frac{m_{2}+u_{*}}{b}\right)^{4}} \\
0
\end{array}\right)
$$

and

$$
\begin{aligned}
& F_{11}=\left(\begin{array}{c}
-\frac{\delta}{u_{*}+m 1 \frac{m_{2}+u_{*}}{b}}+\frac{\delta u_{*}}{\left(u_{*}+m 1 \frac{m_{2}+u_{*}}{b}\right)^{2}}+\frac{\delta m_{1} \frac{m_{2}+u_{*}}{b}}{\left(u_{*}+m 1 \frac{m_{2}+u_{*}}{b}\right)^{2}}-\frac{2 \delta m_{1} u_{*} \frac{m_{2}+u_{*}}{b}}{\left(u_{*}+m 1 \frac{m_{2}+u_{*}}{b}\right)^{3}} \\
\frac{2 \alpha_{2 *}}{m_{2}+u_{*}}
\end{array}\right), \\
& F_{21}=\left(\begin{array}{c}
\frac{2 \delta}{\left(u_{*}+m 1 \frac{m_{2}+u_{*}}{b}\right)^{2}}-\frac{2 \delta u_{*}}{\left(u_{*}+m 1 \frac{m_{2}+u_{*}}{b}\right)^{3}}-\frac{4 \delta m_{1} \frac{m_{2}+u_{*}}{b}}{\left(u_{*}+m 1 \frac{m_{2}+u_{*}}{b}\right)^{3}}+\frac{6 \delta m_{1} u_{*} \frac{m_{2}+u_{*}}{b}}{\left(u_{*}+m 1 \frac{m_{2}+u_{*}}{b}\right)^{4}} \\
\frac{-4 \alpha_{2 *}}{\left(m_{2}+u_{*}\right)^{2}}
\end{array}\right), \\
& F_{12}=\left(\begin{array}{c}
\frac{2 \delta}{\left(u_{*}+m 1 \frac{m_{2}+u_{*}}{b}\right)^{2}}-\frac{4 \delta m_{1} u_{*}}{\left(u_{*}+m 1 \frac{m_{2}+u_{*}}{b}\right)^{3}}-\frac{2 \delta m_{1}^{2} \frac{m_{2}+u_{*}}{b}}{\left(u_{*}+m 1 \frac{m_{2}+u_{*}}{b}\right)^{3}}+\frac{6 \delta m_{1}^{2} u_{*} \frac{m_{2}+u_{*}}{b}}{\left(u_{*}+m 1 \frac{m_{2}+u_{*}}{b}\right)^{4}} \\
\frac{2 \alpha_{2 *} b}{\left(m_{2}+u_{*}\right)^{2}}
\end{array}\right) .
\end{aligned}
$$

Moreover, let

$$
\mathcal{M}_{n}(\lambda)=\left(\begin{array}{cc}
\lambda+d_{1}\left(\frac{n}{l}\right)^{2}-\alpha_{1 *} r\left(u_{4}\right) & \alpha_{1 *} g\left(u_{4}\right) \\
-\frac{\alpha_{2 *}}{b} & \lambda+d_{2}\left(\frac{n}{l}\right)^{2}+\alpha_{2 *}
\end{array}\right) .
$$

Then we can obtain that $\xi_{0} \in \mathbb{C}^{2}$ and $\xi_{n_{*}} \in \mathbb{R}^{2}$ are the eigenvectors associated with the eigenvalues $i \omega_{c}\left(\omega_{c}=\sqrt{h_{0}}\right)$ and 0 , respectively, and $\eta_{0} \in \mathbb{C}^{2}$ and $\eta_{n_{*}} \in \mathbb{R}^{2}$ are the corresponding adjoint eigenvectors, where

$$
\begin{aligned}
& \xi_{0}=\frac{1}{\alpha_{1 *} g\left(u_{4}\right)}\left(\begin{array}{c}
\alpha_{1 *} g\left(u_{4}\right) \\
\alpha_{1 *} r\left(u_{4}\right)-i \omega_{c}
\end{array}\right), \\
& \eta_{0}=\frac{1}{2 \omega_{c}}\left(\begin{array}{c}
\omega_{c}-i \alpha_{2 *} \\
i \alpha_{1 *} g\left(u_{4}\right)
\end{array}\right), \\
& \xi_{n_{*}}=\frac{1}{\alpha_{1 *} g\left(u_{4}\right)}\left(\begin{array}{c}
\alpha_{1 *} g\left(u_{4}\right) \\
\alpha_{2 *} g\left(u_{4}\right)-d_{1}\left(\frac{n_{*}}{l}\right)^{2}
\end{array}\right), \\
& \eta_{n_{*}}=\frac{1}{\left(d_{1}+d_{2}\right)\left(\frac{n_{*}}{l}\right)^{2}}\left(\begin{array}{c}
\alpha_{2 *}+d_{2}\left(\frac{n_{*}}{l}\right)^{2} \\
\alpha_{1 *} g\left(u_{4}\right)
\end{array}\right) .
\end{aligned}
$$

Denote

$$
\Phi_{1}=\left(\xi_{0}, \bar{\xi}_{0}\right), \quad \Phi_{2}=\xi_{n_{*}}, \quad \Psi_{1}=\operatorname{col}\left(\eta_{0}^{T}, \bar{\eta}_{0}^{T}\right), \quad \Psi_{2}=\eta_{n_{*}}^{T}
$$

Then we have

$$
\left\langle\Psi_{1}, \Phi_{1}\right\rangle=I_{2}, \quad\left\langle\Psi_{2}, \Phi_{2}\right\rangle=1
$$

where $I_{2}$ is the $2 \times 2$ identity matrix. Thus, the phase space $\mathscr{X}$ for (27) can be decomposed as

$$
\mathscr{X}=\mathcal{P} \oplus \mathcal{Q}, \quad \mathcal{P}=\operatorname{Im} \pi, \quad \mathcal{Q}=\operatorname{Ker} \pi
$$


where $\operatorname{dim} \mathcal{P}=3$ and $\pi: \mathscr{X} \mapsto \mathcal{P}$ is the projection defined by

$$
\pi(U)=\left(\Phi_{1}\left|\Psi_{1},\left(\left[U, \beta_{0}^{(1)}\right],\left[U, \beta_{0}^{(2)}\right]\right)^{T}\right\rangle\right)^{T} \beta_{0}+\left(\Phi_{2}\left\langle\Psi_{2},\left(\left[U, \beta_{n_{*}}^{(1)}\right],\left[U, \beta_{n_{*}}^{(2)}\right]\right)^{T}\right\rangle\right)^{T} \beta_{n_{*}},
$$

with $\beta_{0}=\operatorname{col}\left(\beta_{0}^{(1)}, \beta_{0}^{(2)}\right)$ and $\beta_{n_{*}}=\operatorname{col}\left(\beta_{n_{*}}^{(1)}, \beta_{n_{*}}^{(2)}\right)$.

According to (31), $U \in \mathscr{X}$ can be decomposed as

$$
\begin{aligned}
U & =\left(\Phi_{1}\left(\begin{array}{l}
z_{1} \\
z_{2}
\end{array}\right)\right)^{T}\left(\begin{array}{l}
\beta_{0}^{(1)} \\
\beta_{0}^{(2)}
\end{array}\right)+\left(z_{3} \Phi_{2}\right)^{T}\left(\begin{array}{c}
\beta_{n_{*}}^{(1)} \\
\beta_{n_{*}}^{(2)}
\end{array}\right)+w \\
& =\left(z_{1} \xi_{0}+z_{2} \bar{\xi}_{0}\right) \gamma_{0}(x)+z_{3} \xi_{n_{*}} \gamma_{n_{*}}(x)+\left(\begin{array}{l}
w_{1} \\
w_{2}
\end{array}\right) \\
& =\left(\Phi_{1}, \Phi_{2}\right)\left(\begin{array}{c}
z_{1} \gamma_{0}(x) \\
z_{2} \gamma_{0}(x) \\
z_{3} \gamma_{n_{*}}(x)
\end{array}\right)+\left(\begin{array}{l}
w_{1} \\
w_{2}
\end{array}\right)
\end{aligned}
$$

where

$$
\left(\begin{array}{l}
z_{1} \\
z_{2}
\end{array}\right)=\left\langle\Psi_{1},\left(\left[U, \beta_{0}^{(1)}\right],\left[U, \beta_{0}^{(2)}\right]\right)^{T}\right\rangle, \quad z_{3}=\left\langle\Psi_{2},\left(\left[U, \beta_{n_{*}}^{(1)}\right],\left[U, \beta_{n_{*}}^{(2)}\right]\right)^{T}\right\rangle .
$$

Setting $\Phi=\left(\Phi_{1}, \Phi_{2}\right), z_{x}=\left(z_{1} \gamma_{0}(x), z_{2} \gamma_{0}(x), z_{3} \gamma_{n_{*}}(x)\right)^{T}$, (32) can be transformed into

$$
U=\Phi z_{x}+w
$$

Let

$$
\left(\begin{array}{l}
{\left[\tilde{F}, \beta_{v}^{(1)}\right]} \\
{\left[\tilde{F}, \beta_{v}^{(2)}\right]}
\end{array}\right)_{v=0}^{v=n_{*}}=\operatorname{col}\left(\left(\begin{array}{l}
{\left[\tilde{F}, \beta_{0}^{(1)}\right]} \\
{\left[\tilde{F}, \beta_{0}^{(2)}\right]}
\end{array}\right),\left(\begin{array}{l}
{\left[\tilde{F}, \beta_{n_{*}}^{(1)}\right]} \\
{\left[\tilde{F}, \beta_{n_{*}}^{(2)}\right]}
\end{array}\right)\right)
$$

then, denoting the restriction of $\mathscr{L}$ to $\mathcal{Q}$ by $\mathscr{L}_{1}$, we have

$$
\left\{\begin{array}{l}
\dot{z}=B z+\Psi\left(\left[\begin{array}{c}
{\left[\tilde{F}(z, w, \mu), \beta_{v}^{(1)}\right]} \\
{\left[\tilde{F}(z, w, \mu), \beta_{v}^{(2)}\right.}
\end{array}\right)_{v=0}^{v=n_{*}}\right. \\
\dot{w}=\mathscr{L}_{1}(w)+H(z, w, \mu)
\end{array}\right.
$$

where

$$
\begin{aligned}
& z=\left(z_{1}, z_{2}, z_{3}\right)^{T}, \quad B=\operatorname{diag}\left\{i \omega_{c},-i \omega_{c}, 0\right\}, \quad \Psi=\operatorname{diag}\left\{\Psi_{1}, \Psi_{2}\right\}, \\
& \tilde{F}(z, w, \mu)=\tilde{F}\left(\Phi z_{x}+w, \mu\right)
\end{aligned}
$$

with

$$
\begin{aligned}
H(z, w, \mu)= & \tilde{F}(z, w, \mu)-\left(\left\langle\eta_{0}^{T},\left(\begin{array}{c}
{\left[\tilde{F}(z, w, \mu), \beta_{0}^{(1)}\right]} \\
{\left[\tilde{F}(z, w, \mu), \beta_{0}^{(2)}\right]}
\end{array}\right)\right\rangle \xi_{0}\right. \\
& \left.+\left\langle\bar{\eta}_{0}^{T},\left(\begin{array}{l}
{\left[\tilde{F}(z, w, \mu), \beta_{0}^{(1)}\right]} \\
{\left[\tilde{F}(z, w, \mu), \beta_{0}^{(2)}\right]}
\end{array}\right)\right) \bar{\xi}_{0}\right) \gamma_{0}(x)
\end{aligned}
$$




$$
-\left\langle\bar{\eta}_{n_{*}}^{T},\left(\begin{array}{l}
{\left[\tilde{F}(z, w, \mu), \beta_{n_{*}}^{(1)}\right]} \\
{\left[\tilde{F}(z, w, \mu), \beta_{n_{*}}^{(2)}\right]}
\end{array}\right)\right\rangle \bar{\xi}_{n_{*}} \gamma_{n_{*}}(x) .
$$

By a recursive transformation, we can obtain that the normal forms for Turing-Hopf bifurcation are

$$
\dot{z}=B z+\left(\begin{array}{l}
B_{11} \mu_{1} z_{1}+B_{22} \mu_{2} z_{1} \\
\bar{B}_{11} \mu_{1} z_{1}+\bar{B}_{22} \mu_{2} z_{1} \\
B_{13} \mu_{1} z_{3}+B_{23} \mu_{2} z_{3}
\end{array}\right)+\left(\begin{array}{l}
B_{210} z_{1}^{2} z_{2}+B_{102} z_{1} z_{3}^{2} \\
\bar{B}_{210} z_{1}^{2} z_{2}+\bar{B}_{102} z_{1} z_{3}^{2} \\
B_{111} z_{1} z_{2} z_{3}+B_{003} z_{3}^{3}
\end{array}\right)+O\left(|z||\mu|^{2}\right)
$$

where

$$
\begin{aligned}
& B_{11}=\eta_{0}^{T} L_{1}^{(1,0)}\left(\xi_{0}\right)=\frac{i \omega_{c}+\alpha_{2 *}}{2 \alpha_{1 *}}, \\
& B_{21}=\eta_{0}^{T} L_{1}^{(0,1)}\left(\xi_{0}\right)=-\frac{1}{2}-\frac{i \alpha_{1 *} r\left(u_{*}\right)}{2 \omega_{c}}+\frac{i \alpha_{1 *} g\left(u_{*}\right)}{2 b}, \\
& B_{13}=\eta_{n_{*}}^{T} L_{1}^{(1,0)}\left(\xi_{n_{*}}\right)=\frac{d_{1} d_{2}\left(\frac{n_{*}}{l}\right)^{2}+d_{1} \alpha_{2 *}}{\alpha_{1 *}\left(d_{1}+d_{2}\right.}, \\
& B_{23}=\eta_{n_{*}}^{T} L_{1}^{(0,1)}\left(\xi_{n_{*}}\right)=-\frac{\alpha_{1 *} g\left(u_{*}\right)}{b\left(d_{1}+d_{2}\right)\left(\frac{n_{*}}{l}\right)^{2}}+\frac{\alpha_{1 *} r\left(u_{*}\right)}{\left(d_{1}+d_{2}\right)\left(\frac{n_{*}}{l}\right)^{2}}-\frac{d_{1}}{d_{1}+d_{2}},
\end{aligned}
$$

and

$$
\begin{array}{ll}
B_{210}=C_{210}+\frac{3}{2}\left(D_{210}+E_{210}\right), & B_{102}=C_{102}+\frac{3}{2}\left(D_{102}+E_{102}\right), \\
B_{111}=C_{111}+\frac{3}{2}\left(D_{111}+E_{111}\right), & B_{003}=C_{003}+\frac{3}{2}\left(D_{003}+E_{003}\right) .
\end{array}
$$

Moreover, we can obtain

$$
\begin{aligned}
& A_{200}=F_{20}+2 \xi_{02} F_{11}+\xi_{02}^{2} F_{02}=\bar{A}_{020}, \\
& A_{002}=F_{20}+2 \xi_{n_{*} 2} F_{11}+\xi_{n_{*} 2}^{2} F_{02}, \\
& A_{110}=2\left(F_{20}+2 \operatorname{Re}\left(\xi_{02}\right) F_{11}+\left|\xi_{02}\right|^{2} F_{02}\right), \\
& A_{101}=2\left(F_{20}+\left(\xi_{02}+\xi_{n_{*}}\right) F_{11}+\xi_{02} \xi_{n_{*} 2} F_{02}\right)=\bar{A}_{011}, \\
& A_{210}=3\left(F_{30}+F_{03}\left|\xi_{02}\right|^{2} \xi_{02}+\left(2 \xi_{02}+\bar{\xi}_{02}\right) F_{21}+\left(\xi_{02}^{2}+2\left|\xi_{02}\right|^{2}\right) F_{12}\right), \\
& A_{102}=3\left(F_{30}+F_{03}\left|\xi_{n_{*} 2}\right|^{2} \xi_{02}+\left(\xi_{02}+2 \xi_{n_{*} 2}\right) F_{21}+\left(\xi_{n_{*} 2}^{2}+2 \xi_{02} \xi_{n_{*} 2}\right) F_{12}\right), \\
& A_{111}=6\left(F_{30}+F_{03}\left|\xi_{02}\right|^{2} \xi_{n_{*} 2}+\left(\xi_{n_{*} 2}+2 \operatorname{Re} \xi_{02}\right) F_{21}+\left(\left|\xi_{02}\right|^{2}+2 \xi_{n_{*} 2} \operatorname{Re} \xi_{02}\right) F_{12}\right), \\
& A_{003}=F_{30}+F_{03} \xi_{n_{*} 2}^{3}+3\left(\xi_{n_{*} 2} F_{21}+\xi_{n_{*} 2}^{2} F_{12}\right),
\end{aligned}
$$

and

$$
\begin{aligned}
& h_{0200}=\frac{1}{\sqrt{l \pi}}\left(\mathcal{M}_{0}\left(2 i \omega_{c}\right)\right)^{-1}\left(A_{200}-\left(\eta_{0}^{T} A_{200} \xi_{0}+\bar{\eta}_{0}^{T} A_{200} \bar{\xi}_{0}\right)\right), \\
& h_{0020}=\frac{1}{\sqrt{l \pi}}\left(\mathcal{M}_{0}\left(-2 i \omega_{c}\right)\right)^{-1}\left(A_{020}-\left(\eta_{0}^{T} A_{020} \xi_{0}+\bar{\eta}_{0}^{T} A_{020} \bar{\xi}_{0}\right)\right),
\end{aligned}
$$




$$
\begin{aligned}
& h_{0002}=\frac{1}{\sqrt{l \pi}}\left(\mathcal{M}_{0}(0)\right)^{-1}\left(A_{002}-\left(\eta_{0}^{T} A_{002} \xi_{0}+\bar{\eta}_{0}^{T} A_{002} \bar{\xi}_{0}\right)\right), \\
& h_{0110}=\frac{1}{\sqrt{l \pi}}\left(\mathcal{M}_{0}(0)\right)^{-1}\left(A_{110}-\left(\eta_{0}^{T} A_{110} \xi_{0}+\bar{\eta}_{0}^{T} A_{110} \bar{\xi}_{0}\right)\right), \\
& h_{n_{*} 101}=\frac{1}{\sqrt{l \pi}}\left(\mathcal{M}_{n_{*}}\left(i \omega_{c}\right)\right)^{-1}\left(A_{101}-\eta_{n_{*}}^{T} A_{101} \xi_{n_{*}}\right), \\
& h_{n_{*} 011}=\frac{1}{\sqrt{l \pi}}\left(\mathcal{M}_{n_{*}}\left(-i \omega_{c}\right)\right)^{-1}\left(A_{011}-\eta_{n_{*}}^{T} A_{011} \xi_{n_{*}}\right), \\
& h_{\left(2 n_{*}\right) 002}=\frac{1}{\sqrt{2 l \pi}}\left(\mathcal{M}_{2 n_{*}}(0)\right)^{-1} A_{002}, \\
& h_{\left(2 n_{*}\right) 110}=(0,0)^{T} .
\end{aligned}
$$

From [26], we have the following expressions of $C_{i j k}, D_{i j k}$, and $E_{i j k}$ :

$$
\begin{aligned}
& C_{210}=\frac{1}{6 l \pi} \eta_{0}^{T} A_{210}, \quad C_{102}=\frac{1}{6 l \pi} \eta_{0}^{T} A_{102}, \\
& C_{111}=\frac{1}{6 l \pi} \eta_{n_{*}}^{T} A_{111}, \quad C_{003}=\frac{1}{4 l \pi} \eta_{n_{*}}^{T} A_{003}, \\
& D_{210}=\frac{1}{6 l \pi \omega_{c} i}\left(-\left(\eta_{0}^{T} A_{200}\right)\left(\eta_{0}^{T} A_{110}\right)+\left|\eta_{0}^{T} A_{110}\right|^{2}+\frac{2}{3}\left|\eta_{0}^{T} A_{020}\right|^{2}\right) \text {, } \\
& D_{102}=\frac{1}{6 l \pi \omega_{c} i}\left(-2\left(\eta_{0}^{T} A_{200}\right)\left(\eta_{0}^{T} A_{002}\right)+\left(\eta_{0}^{T} A_{110}\right)\left(\bar{\eta}_{0}^{T} A_{002}\right)+2\left(\eta_{0}^{T} A_{002}\right)\left(\eta_{n_{*}}^{T} A_{101}\right)\right), \\
& D_{111}=-\frac{1}{3 l \pi \omega_{c}} \operatorname{Im}\left(\left(\eta_{n_{*}}^{T} A_{101}\right)\left(\eta_{0}^{T} A_{110}\right)\right) \\
& D_{003}=-\frac{1}{3 l \pi \omega_{c}} \operatorname{Im}\left(\left(\eta_{n_{*}}^{T} A_{101}\right)\left(\eta_{0}^{T} A_{002}\right)\right) \\
& E_{210}=\frac{1}{3 \sqrt{l \pi}} \eta_{0}^{T}\left(\left(F_{20}+\xi_{02} F_{11}\right) h_{0110}^{(1)}+\left(\xi_{02} F_{02}+F_{11}\right) h_{0110}^{(2)}\right. \\
& \left.+\left(F_{20}+\bar{\xi}_{02} F_{11}\right) h_{0200}^{(1)}+\left(F_{11}+\bar{\xi}_{02} F_{02}\right) h_{0200}^{(2)}\right), \\
& E_{102}=\frac{1}{3 \sqrt{l \pi}} \eta_{0}^{T}\left(\left(F_{20}+\xi_{02} F_{11}\right) h_{0002}^{(1)}+\left(\xi_{02} F_{02}+F_{11}\right) h_{0002}^{(2)}\right. \\
& \left.+\left(F_{20}+\xi_{n_{*} 2} F_{11}\right) h_{n_{*} 101}^{(1)}+\left(F_{11}+\xi_{n_{*} 2} F_{02}\right) h_{n_{*} 101}^{(2)}\right), \\
& E_{111}=\frac{1}{3 \sqrt{l \pi}} \eta_{n_{*}}^{T}\left(\left(F_{20}+\xi_{02} F_{11}\right) h_{n_{*} 011}^{(1)}+\left(\xi_{02} F_{02}+F_{11}\right) h_{n_{*} 011}^{(2)}\right. \\
& \left.\left.+\left(F_{20}+\bar{\xi}_{02} F_{11}\right) h_{n_{*} 101}^{(1)}+\left(F_{11}+\bar{\xi}_{02} F_{02}\right) h_{n_{*} 101}^{(2)}\right)\right) \\
& +\eta_{n_{*}}^{T}\left(\left(F_{20}+\xi_{n_{*} 2} F_{11}\right)\left(\frac{1}{3 \sqrt{l \pi}} h_{0110}^{(1)}+\frac{1}{3 \sqrt{2 l \pi}} h_{\left(2 n_{*}\right) 110}^{(1)}\right)\right. \\
& \left.+\left(F_{11}+\xi_{n_{*} 2} F_{02}\right)\left(\frac{1}{3 \sqrt{l \pi}} h_{0110}^{(2)}+\frac{1}{3 \sqrt{2 l \pi}} h_{\left(2 n_{*}\right) 110}^{(2)}\right)\right) \text {, } \\
& E_{003}=\eta_{n_{*}}^{T}\left(\left(F_{20}+\xi_{n_{*} 2} F_{11}\right)\left(\frac{1}{3 \sqrt{l \pi}} h_{0002}^{(1)}+\frac{1}{3 \sqrt{2 l \pi}} h_{\left(2 n_{*}\right) 002}^{(1)}\right)\right. \\
& \left.+\left(F_{11}+\xi_{n_{*} 2} F_{02}\right)\left(\frac{1}{3 \sqrt{l \pi}} h_{0002}^{(2)}+\frac{1}{3 \sqrt{2 l \pi}} h_{\left(2 n_{*}\right) 002}^{(2)}\right)\right) \text {. }
\end{aligned}
$$




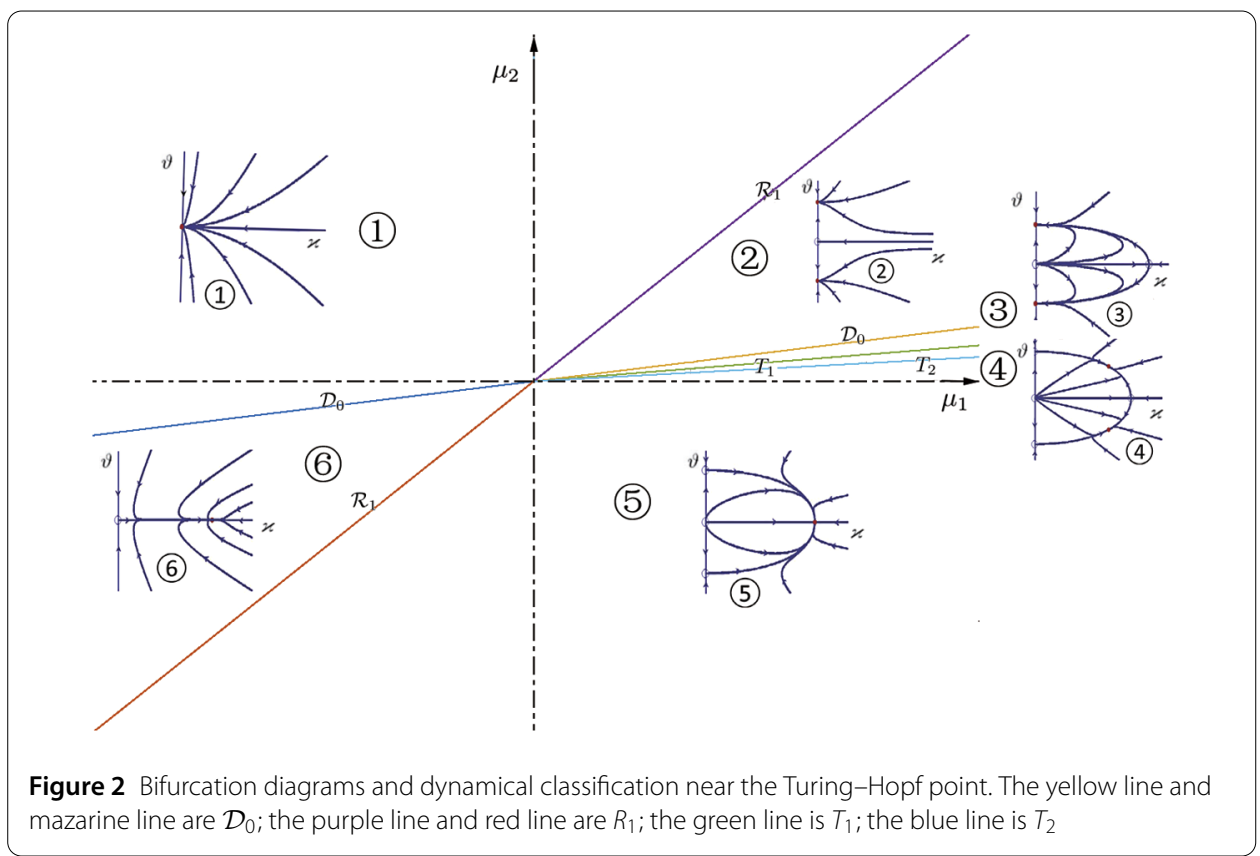

Let $z_{1}=v_{1}-i v_{2}, z_{2}=v_{1}+i v_{2}, z_{3}=v_{3}$, then the normal forms Eq. (37) can be written in real coordinates $v$. Moreover, let $v_{1}=\varkappa \cos \Theta, v_{2}=\varkappa \sin \Theta, v_{3}=\vartheta$, we can rewrite the normal forms from real coordinates to cylindrical coordinates. Finally, removing the azimuthal term and truncating at third order terms, the normal form is as follows:

$$
\begin{aligned}
& \dot{\varkappa}=\alpha_{1}(\mu) \varkappa+\kappa_{11} \varkappa^{3}+\kappa_{12} \varkappa \vartheta^{2}, \\
& \dot{\vartheta}=\alpha_{2}(\mu) \vartheta+\kappa_{21} \varkappa^{2} \vartheta+\kappa_{22} \vartheta^{3},
\end{aligned}
$$

where

$$
\begin{aligned}
& \alpha_{1}(\mu)=\operatorname{Re}\left(B_{11}\right) \mu_{1}+\operatorname{Re}\left(B_{21}\right) \mu_{2}, \quad \alpha_{2}(\mu)=B_{13} \mu_{1}+B_{23} \mu_{2}, \\
& \kappa_{11}=\operatorname{Re}\left(B_{210}\right), \quad \kappa_{12}=\operatorname{Re}\left(B_{102}\right), \quad \kappa_{21}=B_{111}, \kappa_{22}=B_{003} .
\end{aligned}
$$

It is easy to know that truncated normal form (38) is equivalent to the four-dimensional smooth ODE system with Hopf-Hopf bifurcation in [30]. If $\kappa_{11} \kappa_{22}>0$ holds, we know that the dynamics of system (23) is topologically equal to normal forms (38) at the neighborhood of the bifurcation point.

\section{Numerical simulations}

In this section, we verify the theoretical analysis by numerical simulations. Let $\Omega=(0, \pi)$ and choose the following parameters:

$$
\begin{array}{ll}
d_{1}=0.02, & d_{2}=0.35, \quad b=0.35, \quad \delta=0.6, \\
m_{1}=0.5, \quad m_{2}=0.3, \quad h=0.3 .
\end{array}
$$

Then one can check that system (23) has a positive steady state $E_{4}(0.6115,2.6044)$ and $r\left(u_{4}\right)=0.0775>0, \frac{g\left(u_{4}\right)}{b}-r\left(u_{4}\right)=0.0976>0, \Lambda=0.00044>0$. From Theorem 3.4(iii), 

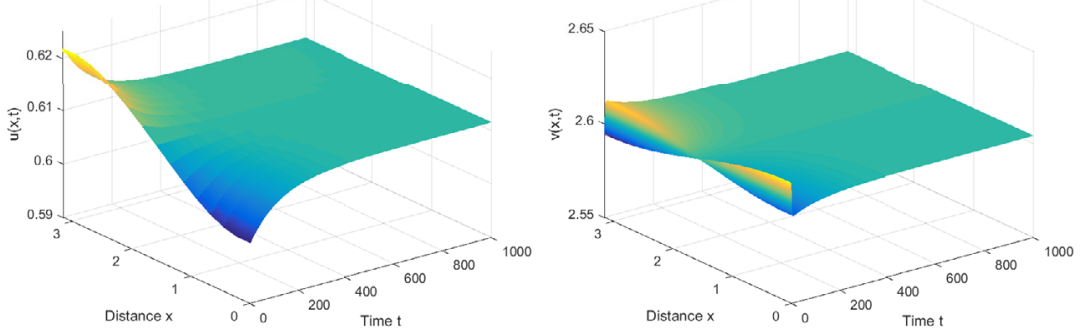

Figure 3 When $\left(\mu_{1}, \mu_{2}\right)=(0.22,0.132)$ lies in region (1), the positive constant steady state $E_{4}(0.6115,2.6044)$ is asymptotically stable, where $u(x, 0)=0.6115-0.01 \cos x, v(x, 0)=2.6044+0.01 \cos x$

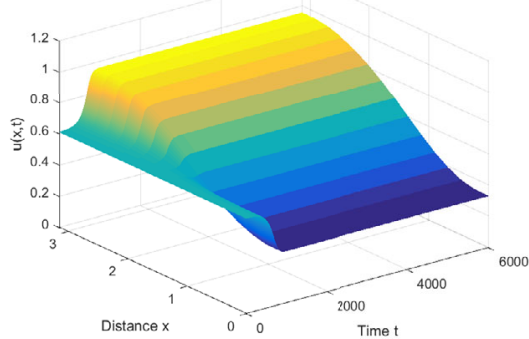

(a)

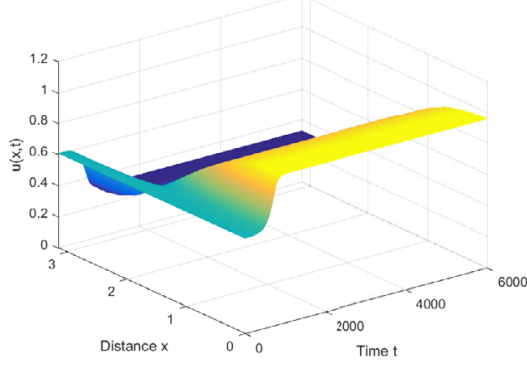

(c)

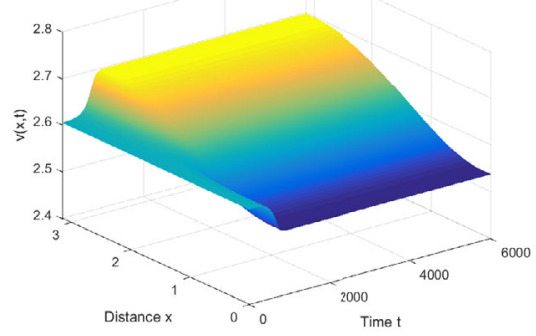

(b)

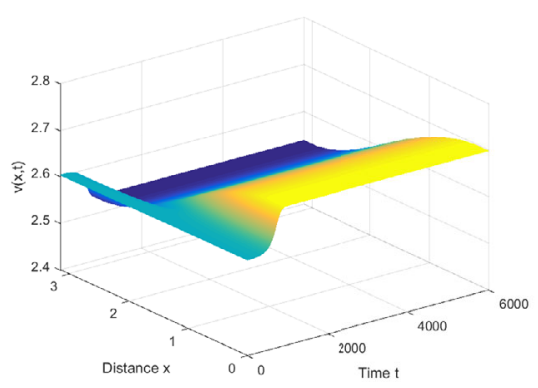

(d)

Figure 4 When $\left(\mu_{1}, \mu_{2}\right)=(0.2,0.016)$ lies in region (2), system (23) has two stable nonconstant steady states, where $u(x, 0)=0.6115-0.001 \cos x, v(x, 0)=2.6044-0.001 \cos x$ in $(\mathbf{a})$ and $(\mathbf{b})$ and $u(x, 0)=0.6115-0.001 \cos x, v(x, 0)=2.6044-0.001 \cos x$ in $(\mathbf{c})$ and $(\mathbf{d})$

we know that system (23) undergoes Turing-Hopf bifurcation at $P^{*}\left(\mathcal{A}_{1}^{-}, r\left(u_{4}\right) \mathcal{A}_{1}^{-}\right)=$ $(0.1388,0.0705)$ (see Fig. 1(a)) and

$$
\mathcal{D}_{0}\left(\alpha_{1}\right): \alpha_{2}=0.0775 \alpha_{1} ; \quad \mathcal{R}_{1}\left(\alpha_{1}\right): \alpha_{2}=\frac{0.0271 \alpha_{1}-0.007}{0.02+0.0976 \alpha_{1}}
$$

Setting $n_{*}=1$, the normal forms truncated to the third order terms are

$$
\begin{aligned}
& \dot{\varkappa}=\left(0.0387 \mu_{1}-0.5 \mu_{2}\right) \varkappa-0.0028 \varkappa^{3}-0.0092 \varkappa \vartheta^{2}, \\
& \dot{\vartheta}=\left(0.0671 \mu_{1}-0.1333 \mu_{2}\right) \vartheta-0.0081 \varkappa^{2} \vartheta-0.0421 \vartheta^{3} .
\end{aligned}
$$




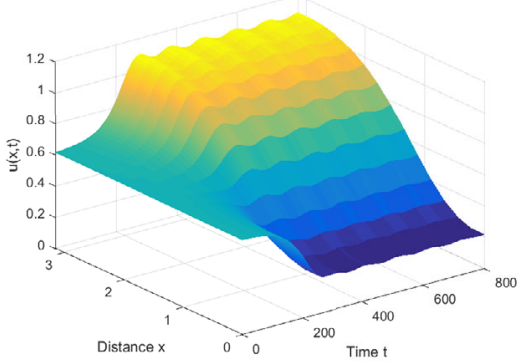

(a)

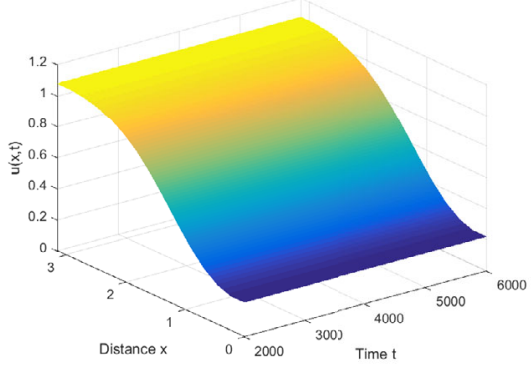

(c)

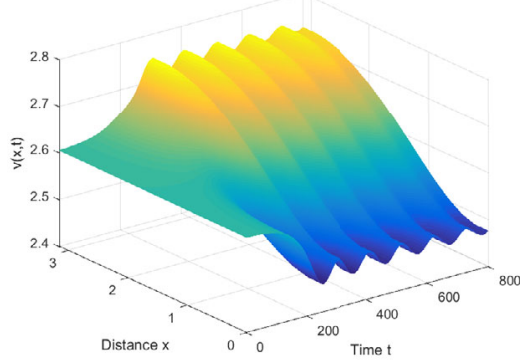

(b)

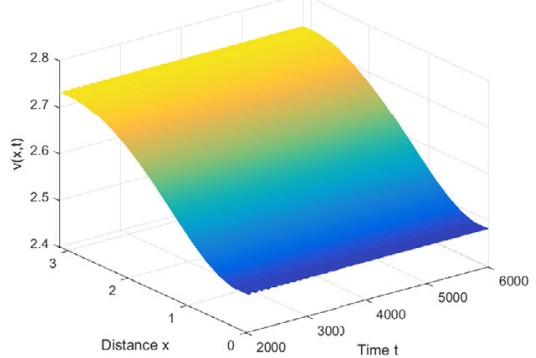

(d)

Figure 5 When $\left(\mu_{1}, \mu_{2}\right)=(0.4,0.0204)$ lies in region (3), system (23) has an unstable constant steady state $E_{4}(0.6115,2.6044)$ and a stable nonconstant steady state, where

$u(x, 0)=0.6115-0.005 \cos x, v(x, 0)=2.6044-0.001 \cos x$. The simulate time is from 0 to 800 in (a) and (b) and the simulate time is from 2000 to 6000 in (c) and (d)

In the parameters plane $\left(\alpha_{1}, \alpha_{2}\right)$, the dynamics of the original system (23) can be equivalent to normal forms system (39) near the Turing-Hopf bifurcation point $P^{*}$. And we have that $\varkappa \geq 0$ and $\vartheta$ is a real number.

There exist a zero equilibrium $p_{0}$ (for all $\mu_{1}$ and $\mu_{2}$ ), three trivial equilibria $p_{1}, p_{2}^{ \pm}$, and two nontrivial equilibria $p_{3}^{ \pm}$for system (39):

$$
\begin{aligned}
& p_{0}=(0,0), \\
& p_{1}=\left(\sqrt{13.9353 \mu_{1}-179.8956 \mu_{2}}, 0\right), \quad \text { for } \mu_{2}<0.0775 \mu_{1}, \\
& p_{2}^{ \pm}=\left(0, \pm \sqrt{1.5948 \mu_{1}-3.1672 \mu_{2}}\right), \quad \text { for } \mu_{2}<0.5035 \mu_{1}, \\
& p_{3}^{ \pm}=\left(\sqrt{23.839 \mu_{1}-467.9284 \mu_{2}}, \pm \sqrt{-2.976 \mu_{1}+86.5523 \mu_{2}}\right)
\end{aligned}
$$

for $\mu_{2}<0.0509 \mu_{1}$ and $\mu_{2}<0.0344 \mu_{1}$.

The critical bifurcation lines are the following:

$$
\begin{aligned}
& \mathcal{D}_{0}: \mu_{2}=0.0775 \mu_{1} ; \quad \mathcal{R}_{1}: \mu_{2}=0.5035 \mu_{1} ; \\
& T_{1}: \mu_{2}=0.0509 \mu_{1}, \quad \mu_{2}>0 ; \quad T_{2}: \mu_{2}=0.0344 \mu_{1}, \quad \mu_{2}>0 .
\end{aligned}
$$

Then we can draw the bifurcation diagram in the $\left(\mu_{1}, \mu_{2}\right)$ parameter space (see Fig. 2). There are four straight lines $\mathcal{D}_{0}, \mathcal{R}_{1}, T_{1}, T_{2}$, and the $\left(\mu_{1}, \mu_{2}\right)$ plane is divided into six regions marked as (1)-6). 


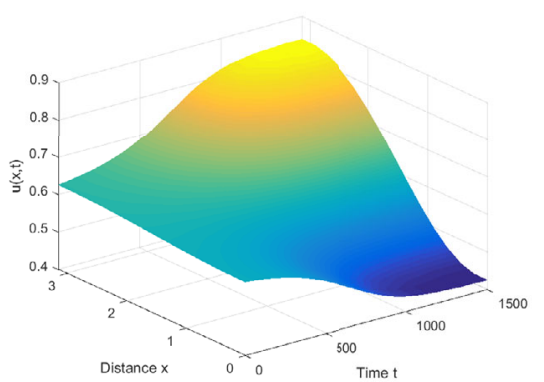

(a)

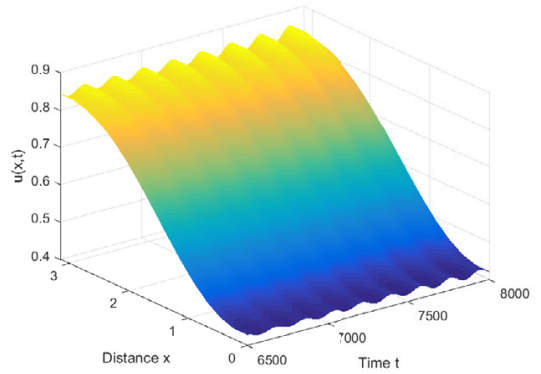

(c)

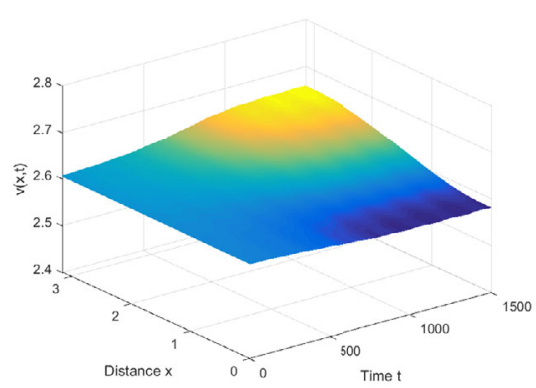

(b)

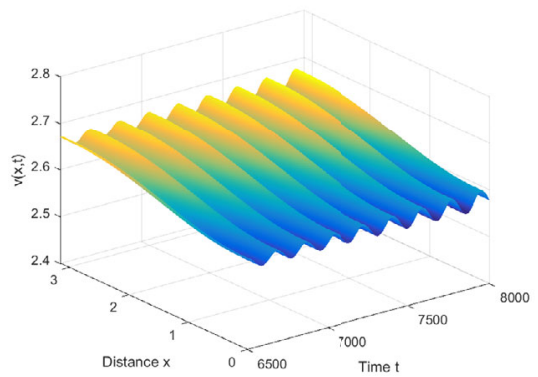

(d)

Figure 6 When $\left(\mu_{1}, \mu_{2}\right)=(0.1,0.005)$ lies in region (4), system (23) has an unstable constant steady state $E_{4}(0.6115,2.6044)$ and a stable spatially inhomogeneous periodic solution, where $u(x, 0)=0.6115-0.015 \cos x, v(x, 0)=2.6044-0.001 \cos x$. The simulate time is from 0 to 1500 in (a) and $(\mathbf{b})$ and the simulate time is from 6500 to 8000 in (c) and (d)

In region (1), system (39) has an asymptotically stable equilibrium $p_{0}$. So system (23) has an asymptotically stable constant steady state (see Fig. 3). In region (2), system (39) has an unstable equilibrium $p_{0}$ and two asymptotically stable equilibria $p_{2}^{ \pm}$. Thus, system (23) has two stable nonconstant steady states (see Fig. 4). In region (3, system (39) has two unstable equilibria $p_{0}, p_{1}$ and two asymptotically stable equilibria $p_{2}^{ \pm}$. Hence, system (23) has two stable nonconstant steady states (see Fig. 5). In region (4), system (39) has four unstable equilibria $p_{0}, p_{1}, p_{2}^{ \pm}$and two asymptotically stable equilibria $p_{3}^{ \pm}$. It follows that system (23) has two stable spatially inhomogeneous periodic solutions (see Fig. 6). In region (5), system (39) has three unstable equilibria $p_{0}, p_{2}^{ \pm}$and an asymptotically stable equilibrium $p_{1}$. Therefore, system (23) has a stable spatially homogeneous periodic solution (see Fig. 7). In region (6), system (39) has an unstable equilibrium $p_{0}$ and an asymptotically stable equilibrium $p_{1}$, which implies that system (23) has a stable spatially homogeneous periodic solution (see Fig. 8).

\section{Acknowledgements}

The authors are very grateful to the referees for their valuable suggestions.

Funding

This work is supported by the Fundamental Research Funds for the Central Universities (No. 2572016CB08).

Availability of data and materials

Not applicable.

Ethics approval and consent to participate

Not applicable. 


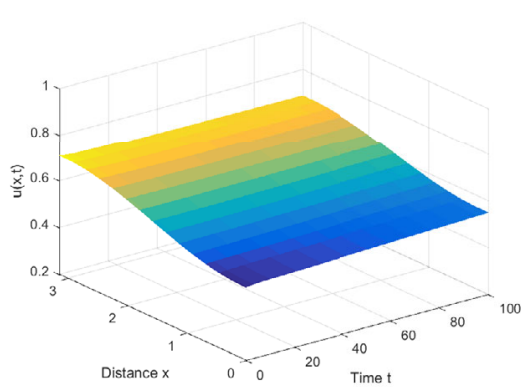

(a)

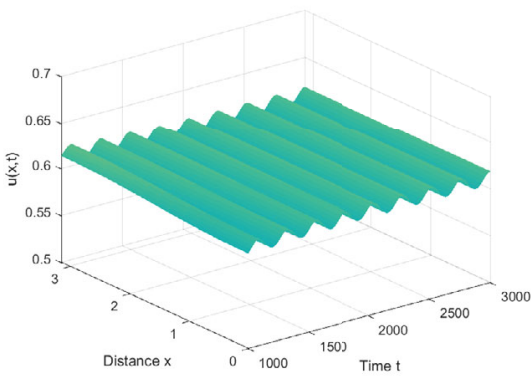

(c)

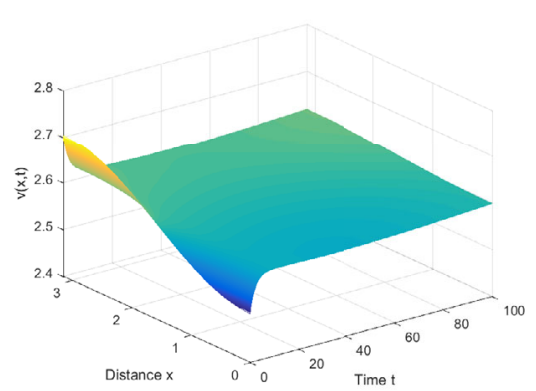

(b)

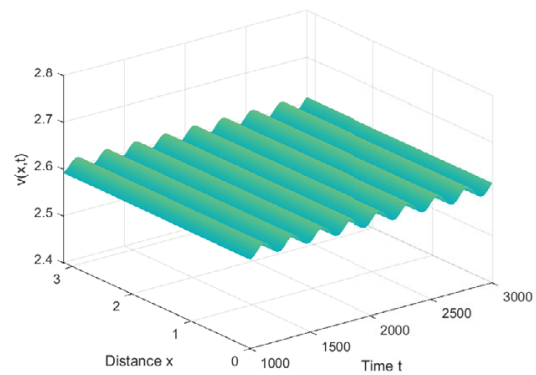

(d)

Figure 7 When $\left(\mu_{1}, \mu_{2}\right)=(0.001,0.00002)$ lies in region (5), system (23) has an unstable constant steady state $E_{4}(0.6115,2.6044)$ and a stable spatially homogeneous periodic solution, where $u(x, 0)=0.6115-0.1 \cos x, v(x, 0)=2.6044-0.1 \cos x$. The simulate time is from 0 to 100 in $(\mathbf{a})$ and $(\mathbf{b})$ and the simulate time is from 1000 to 3000 in (c) and (d)
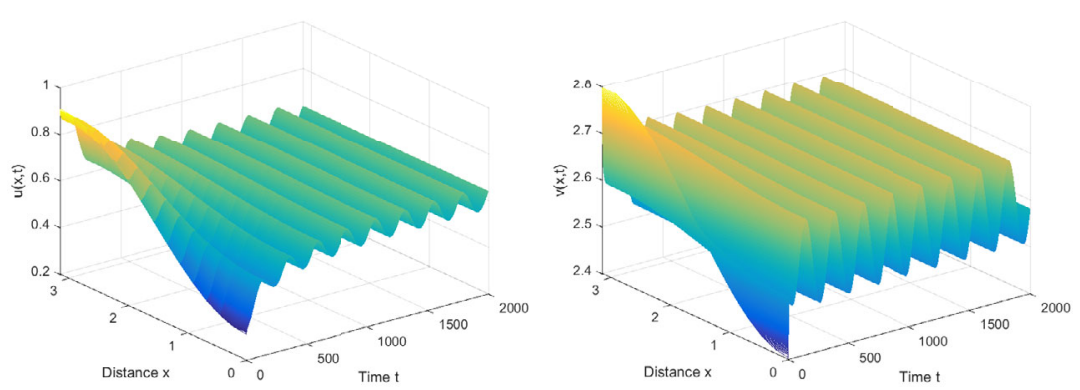

Figure 8 When $\left(\mu_{1}, \mu_{2}\right)=(-0.0001,-0.0002)$ lies in region (6), system (23) has a stable spatially homogeneous periodic solution, where $u(x, 0)=0.6115-0.3 \cos x, v(x, 0)=2.6044-0.2 \cos x$

\section{Competing interests}

The authors declare that they have no competing interests.

\section{Consent for publication}

Not applicable.

\section{Authors' contributions}

All authors participated in the writing and coordination of the manuscript and all authors read and approved the final manuscript.

\section{Publisher's Note}

Springer Nature remains neutral with regard to jurisdictional claims in published maps and institutional affiliations. 
Received: 19 November 2018 Accepted: 29 April 2019 Published online: 02 August 2019

\section{References}

1. Yi, F., Wei, J., Shi, J.: Bifurcation and spatiotemporal patterns in a homogeneous diffusive predator-prey system. J. Differ. Equ. 246, 1944-1977 (2009)

2. Wang, J., Shi, J., Wei, J.: Dynamics and pattern formation in a diffusive predator-prey system with strong Allee effect in prey. J. Differ. Equ. 251, 1276-1304 (2011)

3. Yang, R., Wei, J.: Bifurcation analysis of a diffusive predator-prey system with nonconstant death rate and Holling III functional response. Chaos Solitons Fractals 70, 1-13 (2015)

4. Yan, X., Zhang, C.: Stability and Turing instability in a diffusive predator-prey system with Beddington-DeAngelis functional response. Nonlinear Anal., Real World Appl. 20,1-13 (2014)

5. Tang, X., Song, Y.: Stability, Hopf bifurcations and spatial patterns in a delayed diffusive predator-prey model with herd behavior. Appl. Math. Comput. 254, 375-391 (2015)

6. Sun, G.: Mathematical modeling of population dynamics with Allee effect. Nonlinear Dyn. 85(1), 1-12 (2016)

7. Song, Y., Zhou, X.: Bifurcation analysis of a diffusive ratio-dependent predator-prey model. Nonlinear Dyn. 78(1), 49-70 (2014)

8. Briggs, C.J., Hoopes, M.F.: Stabilizing effects in spatial parasitoid-host and predator-prey models: a review. Theor. Popul. Biol. 65, 299-315 (2004)

9. Wang, J., Jiang, W.: Bifurcation and chaos of a delayed predator-prey model with dormancy of predators. Nonlinear Dyn. 69(4), 1541-1558 (2012)

10. Hu, D., Cao, H.: Bifurcation and chaos in a discrete-time predator-prey system of Holling and Leslie type. Commun. Nonlinear Sci. Numer. Simul. 22, 702-715 (2015)

11. Zhang, H., Huang, T., Dai, L.: Nonlinear dynamic analysis and characteristics diagnosis of seasonally perturbed predator-prey systems. Commun. Nonlinear Sci. Numer. Simul. 22, 407-419 (2015)

12. Huang, T., Zhang, H., Yang, H., Wang, N., Zhang, F.: Complex patterns in a space- and time-discrete predator-prey model with Beddington-DeAngelis functional response. Commun. Nonlinear Sci. Numer. Simul. 43, 182-199 (2017)

13. Liang, I., Pan, H.: Qualitative analysis of a ratio-dependent Holling-Tanner model. J. Math. Anal. Appl. 34, 954-964 (2007)

14. Banerjee, M., Petrovskii, S.: Self-organised spatial patterns and chaos in a ratio-dependent predator-prey system. Theor. Ecol. 4, 37-53 (2011)

15. Baurmann, M., Gross, T., Feudel, U.: Instabilities in spatially extended predator-prey systems: spatio-temporal patterns in the neighborhood of Turing-Hopf bifurcations. J. Theor. Biol. 245, 220-229 (2007)

16. Shi, H.B., Ruan, S.G., Su, Y., Zhang, J.: Spatiotemporal dynamics of a diffusive Leslie-Gower predator-prey model with ratio-dependent functional response. Int. J. Bifurc. Chaos 25, 1530014 (2015)

17. Zhang, L., Liu, J., Banerjee, M.: Hopf and steady state bifurcation analysis in a ratio-dependent predator-prey model. Commun. Nonlinear Sci. Numer. Simul. 44, 52-73 (2017)

18. Arditi, R., Ginzburg, L.R.: Coupling in predator-prey dynamics: ratio-dependence. J. Theor. Biol. 139, $311-326$ (1989)

19. Bishop, M.J., Kelaher, B.P., Smith, M., York, P.H., Booth, D.J.: Ratio-dependent response of a temperature Australian estuarine system to sustained nitrogen loading. Oecologia 149, 701-708 (2006)

20. Hanski, l.: The function response of predator: worries about scale. Trees 6, 141-142 (1991)

21. Reeve, J.D.: Predation and bark beetle dynamics. Oecologia 112, 48-54 (1997)

22. Nindjin, A.F., Aziz-Alaoui, M.A., Cadivel, M.: Analysis of a predator-prey model with modified Leslie-Gower and Holling-type II schemes with time delay. Nonlinear Anal., Real World Appl. 7, 1104-1118 (2006)

23. Upadhyay, R.K., Rai, V.: Crisis-limited chaotic dynamics in ecological systems. Chaos Solitons Fractals 12, 205-218 (2001)

24. Upadhyay, R.K., Lyengar, S.R.K.: Effect of seasonality on the dynamics of 2 and 3 species prey-predator system. Nonlinear Anal., Real World Appl. 6, 509-530 (2005)

25. Faria, T.: Normal forms and Hopf bifurcation for partial differential equations with delay. Trans. Am. Math. Soc. 352 , 2217-2238 (2000)

26. Song, Y., Zhang, T., Peng, Y.: Turing-Hopf bifurcation in the reaction-diffusion equations and its applications. Commun. Nonlinear Sci. Numer. Simul. 33, 229-258 (2016)

27. Tang, X., Song, Y., Zhang, T.: Turing-Hopf bifurcation analysis of a predator-prey model with herd behavior and cross-diffusion. Nonlinear Dyn. 86, 73-89 (2016)

28. Yang, R., Song, Y.: Spatial resonance and Turing-Hopf bifurcation in the Gierer-Meinhardt model. Nonlinear Anal., Real World Appl. 31, 356-387 (2016)

29. Xu, X., Wei, J.: Turing-Hopf bifurcation of a class of modified Leslie-Gower model with diffusion. Discrete Contin. Dyn. Syst., Ser. B 32(2), 765-781 (2018)

30. Kuznetsov, Y.A.: Elements of Applied Bifurcation Theory. Springer, New York (1998) 